\title{
Asymptotic Formulae for Lorenz and Horseshoe Knots
}

\author{
Simon Waddington \\ Mathematics Institute, University of Warwick, Coventry, CV4 7AL, U.K.`
}

Received: 10 March 1993

\begin{abstract}
We derive various asymptotic formulae for the numbers of closed orbits in the Lorenz and Horseshoe templates with given knot invariants, (for example braid index and genus). We indicate how these estimates can be applied to more complicated flows by giving a bound for the genus of knotted periodic orbits in the 'figure of eight' template.
\end{abstract}

\section{Introduction}

Let $\Phi_{t}$ be a flow on $S^{3}$ (for example a Lorenz flow or an Axiom A no cycles flow) with countably many periodic orbits $\left(\tau_{n}\right)_{n=1}^{\infty}$. We regard each closed orbit as a knot in $S^{3}$. The set of all infinite collections of knots $\left(K_{n}\right)_{n=1}^{\infty}$ has the cardinality of the continuum. However, for Axiom A no cycle flows for example, the set of such collections of knots which occur as periodic orbits is countable, so only special ones can occur. The central problem in the study of knotted periodic orbits of flows in $S^{3}$ is to classify these families of knots, or more realistically to find restrictions on them.

In his survey lecture [W4], Williams suggested that a useful approach to this problem would be to associate a knot invariant $k i(\tau)$ to each closed orbit $\tau$ (e.g. braid index, genus) and to find restrictions on the sequences $\left(k i\left(\tau_{n}\right)\right)_{n=1}^{\infty}$. In this paper, we provide a solution for three specific examples of flows. For various knot invariants, we show that such sequences must satisfy precise asymptotic formulae or bounds.

In [BW1] and [BW2], Birman and Williams introduced the notion of a template in $S^{3}$, which consists of a branched two manifold, with charts of two specific types, together with an expanding semiflow defined on it. For certain types of flows in $S^{3}$, one can construct a template for the flow in such a way that the periodic orbits of the flow and the semiflow correspond one-to-one, and this correspondence preserves knot types. (Strictly, one may first need to exclude finitely many orbits.) So we will study only knotted periodic orbits in templates.

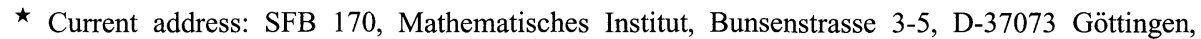
Germany 
First we study the Lorenz attractor. The famous system of differential equations

$$
\begin{aligned}
& x^{\prime}=-10 x+10 y, \\
& y^{\prime}=28-y-x z, \\
& z^{\prime}=-(8 / 3) z+x y
\end{aligned}
$$

of E.N. Lorenz [Lo] has been extensively studied, (see for example [Gu, R]), as it is an important example of a "strange attractor" in the Ruelle-Takens sense, [RT]. This model was used by physicists to study atmospheric convection.

Guckenheimer and Williams [Gu, W1] introduced a mechanism for this attractor which became known as the "geometric Lorenz attractor". Williams [W1] constructed a template for this flow, and jointly with Birman [BW1], attempted to determine which families of knots actually occurred as periodic orbits when the Poincaré map $T$ on the branch line had the form $T \xi=2 \xi(\bmod 1)$.

Our approach is more quantitative. Specifically, we give a precise formula for $\#\{\tau: b(\tau) \leqq m\}$ and (upper and lower) bounds for $\#\{\tau: g(\tau) \leqq m\}$. (Here $b(\tau)$ denotes the braid index and $g(\tau)$ denotes the genus of a generic closed orbit $\tau$.) We also give a precise asymptotic formula for the sum

$$
\sum_{b(\tau) \leqq m} g(\tau)
$$

In all but exceptional cases these numbers are finite, for $m$ fixed. This is the content of Theorems 1-3. In Corollaries 1-4, we include partial results on the degrees of the Alexander and Jones polynomials of closed orbits.

Our results hold for a wide choice of Poincaré maps. We make extensive use of the kneading theory for the Lorenz attractor, developed in [W1]. An essential observation in all our results is that the link of knotted periodic orbits which exist on a given template depends only on the kneading invariants. Alternatively, two Poincare maps with the same kneading invariants have associated semiflows with essentially the same link of knotted periodic orbits.

In Sect. 5, we discuss the special case of centrally symmetric $\beta$-transformations, as described in [P3], to illustrate the effect of renormalisation on the link of Lorenz knots.

Next, we consider a different embedding of the Lorenz template, called the Horseshoe template. In the special case that the Poincare map takes the form

$$
T(\xi)= \begin{cases}2 \xi & \text { for } 0 \leqq \xi \leqq 1 / 2 \\ 2(1-\xi) & \text { for } 1 / 2<\xi \leqq 1\end{cases}
$$

this template has the same link of periodic orbits as the suspension of the well known Smale horseshoe map. We adapt the kneading theory for the Lorenz system, and modify our estimates to give asymptotic bounds on $\#\{\tau: g(\tau) \leqq m\}$. (See Theorem 4.)

Finally, we consider the "Figure of Eight" template, as studied in [BW2]. This arises in the study of the planetary orbits of the figure of eight knot. From a more dynamical viewpoint, this template is determined by taking a suspension flow of the map

$$
\left(\begin{array}{ll}
2 & 1 \\
1 & 1
\end{array}\right)
$$


acting on the punctured torus $\mathbb{T}^{2} \backslash\{0\}$. Using the fact that the figure of eight template contains a composite of two Lorenz templates, we outline how to give a lower bound for $\#\{\tau: g(\tau) \leqq m\}$, using our previous estimates.

\section{Preliminaries}

Let $B_{n}$ denote the Burau braid group, [Bi, p. 5]. By a well known result of Artin, every (tame) knot $K$ in $S^{3}$ can be presented as a closed braid $\hat{b}$, where $b \in B_{n}$ for some $n$. The least such $n$ is called the braid index $b(K)$ of $K$, and is a knot invariant. Another important knot invariant is the genus $g(K)$ of $K$, which is defined to be the minimal genus of any Seifert surface spanning $K$.

We now define the (one-sided) subshifts of finite type. These were first introduced in a purely mathematical context in [P4]. Let $A$ be a $k \times k$, zero-one matrix and suppose $A$ is irreducible, i.e. for each $i, j$, there exists $n$ such that $A^{n}(i, j)>0$.

Let

$$
\Sigma_{A}=\left\{w \in \prod_{n=0}^{\infty}\{1, \ldots, v\}: A\left(w_{n}, w_{n+1}\right)=1, \text { for each } n \geqq 0\right\} .
$$

For fixed $\theta \in(0,1)$, define a metric $\rho_{\theta}$ on $\Sigma_{A}$ by $\rho_{\theta}(x, y)=\theta^{N}$, where $N$ is the largest integer such that $x_{i}=y_{i}$ for $0 \leqq i<N$. The metric space $\left(\Sigma_{A}, \rho_{\theta}\right)$ is compact and zero dimensional. Define the shift $\sigma: \Sigma_{A} \rightarrow \Sigma_{A}$ by $(\sigma x)_{n}=x_{n+1}$, which is a continuous, bounded-to-one map.

The pressure map $P: C\left(\Sigma_{A}\right) \rightarrow \mathbb{R}$ is defined by

$$
P(f)=\sup _{\mu}\left\{h(\mu)+\int f d \mu\right\},
$$

where the supremum is over all $\sigma$-invariant probabilities $\mu$ on $\Sigma_{A}$. $(h(\mu)$ is the entropy of $\sigma$ with respect to $\mu$.) If additionally, we assume that $f$ is Hölder continuous then the supremum is attained uniquely by a measure $m_{f}$. (If $f$ depends on only finitely many coordinates, i.e. for $n$ fixed, $f(x)=f\left(x_{0} x_{1} \ldots x_{n}\right)$ for all $x \in \Sigma_{A}$, then $f$ is Hölder continuous.)

By Abramov's Theorem [A], there is a unique positive real number $\lambda$ such that $P(-\lambda f)=0$. Moreover,

$$
\lambda=\sup _{\mu} \frac{h(\mu)}{\int f d \mu}
$$

and the supremum is uniquely attained by the measure $m_{-\lambda f}$.

We now briefly discuss Markov maps of the unit interval. Let $I=[0,1]$, and let $T: I \rightarrow I$ be piecewise $C^{1}$, where $\left\{c_{i}: i=1, \ldots, r\right\}$ are precisely those points where $T^{\prime}$ is discontinuous. Such a map is called locally onto if for each open $J \subset I$, there exists $m$ such that $\bigcup_{j=0}^{m} T^{j} J=I$. This property was introduced in [P2]. The map $T$ is called Markov if there exists a finite set of points $S=\left\{\xi_{j}: j=0, \ldots, v\right\} \subset I$ with $\xi_{0}=0, \xi_{v}=1$ such that

$$
\left\{\lim _{\xi \downarrow \xi_{0}} T(\xi), \lim _{\xi \uparrow \xi_{v}} T(\xi)\right\} \cup \bigcup_{j=1}^{\nu-1}\left\{\lim _{\xi \downarrow \xi_{j}} T(\xi), \lim _{\xi \uparrow \xi_{j}} T(\xi)\right\} \subseteq S .
$$


For $T$ Markov, let $A$ be a $v \times v$ matrix whose entries are 0 or 1 according to the rules

$$
A(i, j)=\left\{\begin{array}{ll}
1 & \text { if } T\left(\xi_{i-1}, \xi_{i}\right) \supseteq\left(\xi_{j-1}, \xi_{j}\right) \\
0 & \text { if } T\left(\xi_{i-1}, \xi_{i}\right) \cap\left(\xi_{j-1}, \xi_{j}\right)=\emptyset
\end{array} .\right.
$$

(The Markov condition ensures these are the only possibilities.) We will always choose $\theta=\sup \left\{\left|T^{\prime}(x)\right|: x \in I \backslash\left\{c_{i}: i=1, \ldots, r\right\}\right\}^{-1}$. Define a map $\pi: \Sigma_{A} \rightarrow I$ by

$$
\pi(w)=\bigcap_{n=0}^{\infty} T^{-n}\left[\xi_{w_{n}}, \xi_{w_{n+1}}\right] .
$$

Then $\pi$ is a semiconjugacy $\pi \sigma=T \pi$, and $\pi$ is Lipschitz, (by our choice of $\theta$ ). The map $\pi$ is one-to-one, except for a countable set points where it is bounded-to-one.

Notation. Let $f, g: \mathbb{N} \rightarrow \mathbb{R}^{+}$. Write $f(n) \sim g(n)$ as $n \rightarrow \infty$ if $f(n) / g(n) \rightarrow 1$ as $n \rightarrow \infty$, and write $f \gg g$ or $g \ll f$ if

$$
\limsup _{n \rightarrow \infty} \frac{g(n)}{f(n)} \leqq 1
$$

\section{The Lorenz Template}

Let $H_{L}$ denote the branched two manifold model of the Lorenz attractor (cf. [W1]), which we have illustrated in Fig. 2.1.

Let $\Phi_{t}: H_{L} \rightarrow H_{L}$ (for $t \geqq 0$ ) denote a semiflow on $H_{L}$ which is downwardly transverse at the branch line $I$, which we parameterise as $I=[0,1]$. Let $T: I \rightarrow I$ denote the Poincaré map, which fails to be defined only at the point $c \in(0,1)$. (See Fig. 2.2 for a typical example). Let $\rho: I \rightarrow \mathbb{R}^{+}$be the first return time map $\rho(\xi)=\inf \left\{t>0: \Phi_{t}(\xi) \in I\right\}$.

Each closed orbit $\tau$ of $\Phi_{t}$ is a knot in $S^{3}$. Thus $\tau$ has a well defined braid index $b(\tau)$ and genus $g(\tau)$.

We now consider the Poincaré map $T$ in more detail. For $\beta>1$, we say $T: I \rightarrow I$ is in $L_{\beta}$ if

(i) $T$ is $C^{1}$ for all $\xi \neq c$, for some $c \in(0,1)$,

(ii) $\lim _{\xi \uparrow c} T(\xi)=1, \lim _{\xi \downarrow c} T(\xi)=0, T(c)=c$, and

(iii) $T^{\prime}(\xi) \geqq \beta$ for all $\xi \neq c$.

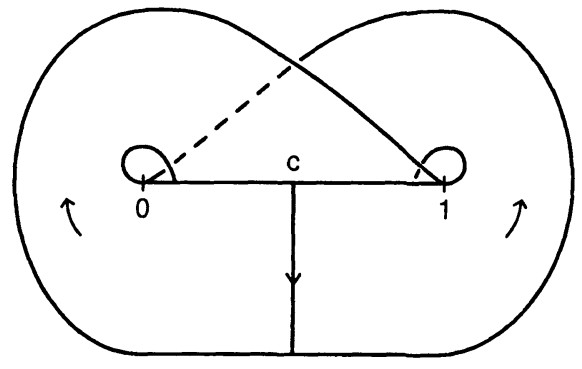

Fig. 2.1.

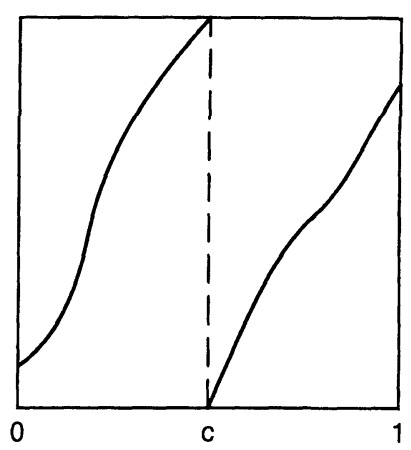

Fig. 2.2. 
A particularly simple class of maps that we will consider are the $\beta$-transformations, which take the form

$$
T(\xi)=T_{\beta, \alpha}(\xi)= \begin{cases}\beta \xi+\alpha(\bmod 1) & \text { if } \xi \neq(1-\alpha) \beta^{-1} \\ (1-\alpha) \beta^{-1} & \text { if } \xi=(1-\alpha) \beta^{-1}\end{cases}
$$

for some $1<\beta \leqq 2, \alpha \geqq 0$ and $\alpha+\beta \leqq 2$.

We now discuss the kneading theory for maps $T \in L_{\beta}$. Let $X_{1}=\prod_{n=0}^{\infty}\{x, y\}$ denote the space of infinite, one-sided sequences of $x$ 's and $y$ 's and let

$$
X_{2}=\bigcup_{m=0}^{\infty}\left(\prod_{i=1}^{m}\{x, y\} \times \prod_{j=m+1}^{\infty}\{0\}\right)
$$

denote all finite (or empty) sequences of $x$ 's and $y$ 's which terminate with infinitely many 0's.

Let $X=X_{1} \cup X_{2}$, and give $X$ the topology induced by the metric

$$
d(u, v)=\sum_{n} \frac{\left|\tilde{u}_{n}-\tilde{v}_{n}\right|}{2^{n}}
$$

where $u=\left(u_{n}\right), v=\left(v_{n}\right)$ and

$$
\tilde{u}_{n}= \begin{cases}-1 & \text { if } u_{n}=x \\ 0 & \text { if } u_{n}=0 \\ 1 & \text { if } u_{n}=y .\end{cases}
$$

Define the shift $\sigma: X \rightarrow X$ by $(\sigma w)_{n}=w_{n+1}$. Let $<$ denote the natural lexicographical ordering on $X$, generated by the ordering $x<0<y$.

A point $w \in X$ is called eventually periodic if there exist finite words, $u, v$ of $x$ 's and $y$ 's such that $w=u \bar{v}$, where $\bar{v}$ denotes the element $v v v \cdots \in X_{1}$. (Here, we allow $u$ to be empty.) Let $Z=X_{2} \cup\left\{w \in X_{1}: w\right\}$ is eventually periodic.

We say that $\kappa=\left(k_{l}, k_{r}\right) \in K$ if $k_{l}, k_{r} \in X$ and

(A1) $k_{l}<k_{r}$, and

(A2) $k_{l} \leqq \sigma^{n} k_{l} \leqq k_{r}$ and $k_{l} \leqq \sigma^{n} k_{r} \leqq k_{r}$ for all $n \geqq 0$.

Define a metric $D$ on $K$ by $D\left(\kappa^{(1)}, \kappa^{(2)}\right)=d\left(k_{l}^{(1)}, k_{r}^{(1)}\right)+d\left(k_{l}^{(2)}, k_{r}^{(2)}\right)$. Let $K^{0}$ denote all $\kappa=\left(k_{l}, k_{r}\right) \in K$ such that $k_{l} \neq \bar{x}$ and $k_{r} \neq \bar{y}$.

Kneading invariants arise in the following way. Let $T \in L_{\beta}$, and for $\xi \in I$, define

and $k_{i}(\xi)=k_{0}\left(T^{i}(\xi)\right)$.

$$
k_{0}(\xi)= \begin{cases}x & \text { if } \xi<c \\ 0 & \text { if } \xi=c \\ y & \text { if } \xi>c\end{cases}
$$

The infinite sequence $k(\xi)=k_{0}(\xi) k_{1}(\xi) k_{2}(\xi) \cdots \in X$ is called the kneading sequence of $\xi \in I$. Moreover, the map $\xi \mapsto k(\xi)$ is strictly monotonic increasing, and the shift $\sigma$ satisfies $\sigma(k(\xi))=k(T(\xi))$. The kneading invariant of $T, \kappa=\kappa_{T}=$ $\left(k_{l}, k_{r}\right) \in K$ is defined to be the pair $(k(0), k(1))$. A sequence $k(\xi)$ is $T$-admissable if and only if for all $m \geqq 0$,

$$
k(0) \leqq \sigma^{m} k(\xi) \leqq k(1)
$$

and either

$$
\sigma^{m} k(\xi)=0, \quad \sigma^{m} k(\xi)<k(1), \quad \text { or } \quad \sigma^{m} k(\xi)>k(0)
$$


In particular, for a finite word $w$ of $x$ 's and $y$ 's, the periodic word $\bar{w}=w w w \cdots$ is allowable if and only if

$$
k(0)<\sigma^{m} w<k(1)
$$

for all $m \geqq 0$.

We say that $\kappa \in K$ is linearly realisable if there exists a $\beta$-transformation with kneading invariant $\kappa$.

Define the trip number $T(w)$ of a finite, aperiodic word $w$ of $x$ 's and $y$ 's to be the number of ' $x y$ ' syllables in $w$, counted cyclically (e.g. $\left.T\left(x^{2} y x y\right)=2\right)$. Suppose that a periodic orbit $\tau$ has kneading sequence $w(\tau)$. Then define the trip number $t(\tau)$ to be $t(\tau)=T(w(\tau))$.

Definition 1, [G]. An element $\kappa=\left(k_{l}, k_{r}\right) \in K$ is called renormalisable if there exist finite words $w_{1}, w_{2}$ of $x$ 's and y's respectively, with respective lengths $N_{1}, N_{2}$ with $N_{1}+N_{2} \geqq 4$ such that $k_{l}=w_{1} w_{2}^{n_{1}} w_{1}^{n_{2}} w_{2}^{n_{3}} \ldots$ and $k_{r}=w_{2} w_{1}^{m_{1}} w_{2}^{m_{2}} w_{1}^{m_{3}} \ldots$ Let the shortest (non-trivial) such choice be $\left(w_{1}^{(1)}, w_{2}^{(1)}\right)$ of lengths $\left(N_{1}^{(1)}, N_{2}^{(1)}\right)$. Then replacing $w_{1}^{(1)}$ by $x$ and $w_{2}^{(1)}$ by $y$, we obtain a renormalised kneading invariant $\kappa^{(1)}$. If this process can be repeated $n$ times, but not $n+1$ times, (using the shortest possible choice at each stage), the kneading invariant is called n-renormalisable. (If $T \in L_{\beta}$ then $n$ is finite, by [G].) If $\kappa$ is not renormalisable, it is called prime.

We give an example of renormalisation in Sect. 6.

Part (i) of the following lemma follows directly from the choice of metric $D$ on $K$. Part (ii) is [W2], Proposition 1.

\section{Lemma 1.}

(i) The set of prime kneading invariants is an open subset of $K$.

(ii) $Z$ is a dense subset of $X$.

We now observe that $\kappa$ is prime is a (necessary and) sufficient condition for $T$ to be locally onto, for any $T \in L_{\beta}$ with kneading invariant $\kappa$.

Lemma 2. ([G], Theorem 2). Let $\kappa_{T} \in K$ be the kneading invariant of $T \in L_{\beta}$. If $\kappa_{T}$ is prime then $T$ is locally onto.

Lemma 3. If $T \in L_{\beta}$ is locally onto and Markov then the transition matrix $A$ is irreducible.

Proof. By hypothesis, $T$ is locally onto, so for each interval $J_{i}=\left(\xi_{i}, \xi_{i+1}\right)$ in the Markov partition, there exists $n_{i}>0$ such that $\bigcup_{j=0}^{n_{l}} T^{j} J_{i}=I$, for $i=1, \ldots, v$. Thus, given $1 \leqq i, l \leqq v$, there exists $j$ such that $J_{l} \cap T^{j} J_{i} \neq \emptyset$, and since $T$ is Markov, $J_{l} \subseteq T^{j} J_{i}$. Thus $A^{j}(i, l) \neq 0$, and since $i, l$ were arbitrary, $A$ is irreducible.

We now prove the existence of a Markov $\beta$-transformation realising certain kneading invariants. This is a modified version of a result in [W2], (but see also $[\mathrm{P} 1])$.

Proposition 1. Let $\kappa=\left(k_{l}, k_{r}\right) \in K$ be prime. Then $\kappa$ is linearly realisable by a map $T \in L_{\beta}$. When additionally, $k_{l}, k_{r} \in Z$, the map $T$ is Markov.

Proof. Let $\kappa=\left(k_{l}, k_{r}\right) \in K$ be prime. First suppose that $k_{l}, k_{r} \in Z$. Then the sets $L=\left\{\sigma^{n} k_{l}: n \geqq 0\right\}$ and $R=\left\{\sigma^{n} k_{r}: n \geqq 0\right\}$ are finite. Let $X^{\prime}=X \cup\left\{0 k_{l}, 0 k_{r}\right\}$ and extend $\sigma$ to a map $X^{\prime} \rightarrow X$. Then let $D=L \cup R \cup\left\{0 k_{l}, 0 k_{r}\right\}$, so that $\sigma D=D$. Write $D=\left\{\eta_{0}, \ldots, \eta_{v}\right\}$, where $\eta_{0}<\eta_{1}<\cdots<\eta_{v}$. 
Let $U \in L_{\beta}$ be any realisation of $\kappa$ and let $\xi_{i} \in I, i=1, \ldots, v$, satisfy $k\left(\xi_{i}\right)=\eta_{i}$. So in particular, since $\xi \mapsto k(\xi)$ is monotonic increasing, $0=\xi_{0}<\xi_{1}<\cdots<$ $\xi_{v}=1, U\left(\left\{\xi_{0}, \ldots, \xi_{v}\right\}\right) \subseteq\left\{\xi_{0}, \ldots, \xi_{v}\right\}$, and $U\left(c^{-}\right)=1, U\left(c^{+}\right)=0$. (Here $k\left(c^{-}\right)=$ $0 k_{r}$ and $k\left(c^{+}\right)=0 k_{l}$.)

Define a $v \times v$, zero-one matrix $A$ by (1.1). By Lemma 2, $U$ is locally onto, and so by Lemma $3, A$ is irreducible. Thus by the Perron Frobenius Theorem for matrices, $A$ has a maximal positive eigenvalue $\lambda$ with positive eigenvector $e=\left(e_{1}, \ldots, e_{v}\right)$, (i.e. $e_{i}>0$, for each $i$ ). Normalise $e$ so that $e_{1}+\cdots+e_{v}=1$. Then choose points $\rho_{0}, \ldots, \rho_{v} \in I$ such that $\rho_{0}=0, \rho_{i}=e_{i}+e_{i-1}+\cdots+e_{2}+e_{1}$ for $i=1, \ldots, v$. Choose $T$ to be the $\beta$-transformation $T \xi=\lambda \xi+\rho_{r}(\bmod 1)$, where $r=\min \{i: A(1, i) \neq 0\}-1$.

Now suppose that at least one of $k_{l}, k_{r}$ is not in $Z$. Since $Z$ is dense in $X$ by Lemma 1(ii), it follows that $(Z \times Z) \cap K$ is dense in $K$. So we may choose a sequence $a^{(n)}=\left(a_{l}^{(n)}, a_{r}^{(n)}\right)$ such that $a^{(n)} \rightarrow \kappa$. By Lemma 1(i), we may assume that each $a^{(n)}$ is prime. Then let $T_{\beta_{n}, \alpha_{n}}$ be the linear realisation of $a^{(n)}$. Let $(\beta, \alpha)$ be a limit point of $\left(\beta_{n}, \alpha_{n}\right)$ in $\mathbb{R}^{2}$. Since the map

$$
\left\{(x, y) \in \mathbb{R}^{2}: 1 \leqq y \leqq 2, x \geqq 0, x+y \leqq 2\right\} \rightarrow K
$$

given by $(x, y) \mapsto \kappa_{T_{y, x}}$ is continuous, $T_{\beta, \alpha}$ is the linear realisation of $\kappa$.

\section{Asymptotics for Braid Index of Lorenz Knots}

In view of Proposition 1, we will now consider a locally onto, $\beta$-transformation $T=T_{\beta, \alpha}$. Let $\kappa_{T} \in K$ denote the kneading invariant of $T$.

Define $f: I \rightarrow I$ to be the characteristic function of the interval $J=\{\xi \in$ $[0, c): T(\xi)>c\}$, or more explicitly,

$$
J=\left(\frac{1-\alpha(1+\beta)}{\beta^{2}}, \frac{1-\alpha}{\beta}\right) .
$$

Also, let $f^{N}=f+f \circ T+f \circ T^{2}+\cdots+f \circ T^{N-1}$.

The following lemma is elementary.

Lemma 4. If $\kappa_{T} \in K^{0}$ then there exists $N>0$ such that $f^{N} \geqq 1$.

Remark 1. The condition $\kappa_{T} \in K^{0}$ may be replaced by the more qualitative assumption that $T$ has no sources. (A point $z \in I$ is called a source if there exists a non-empty open neighbourhood $V$ of $z$ such that $\{z\}=\bigcap_{n \geqq 0} T^{-n} V$.)

For the next definition, we assume further that $T$ is Markov with transition matrix $A$.

Definition 2. [W3] (i) For each sequence $i_{1}, \ldots, i_{r}, \quad(r \geqq 2)$ of distinct $i_{j} \in$ $\{1,2, \ldots, v\}$ such that the product

$$
A\left(i_{1}, i_{2}\right) A\left(i_{2}, i_{3}\right) \cdots A\left(i_{r}, i_{1}\right) \neq 0
$$

let $\left(i_{1}, i_{2}, \ldots, i_{r}\right)$ be the equivalence class under cyclic permutations of this $r$-tuple. These equivalence classes are called free knot symbols and the indices $i_{1}, i_{2}, \ldots, i_{r}$ are called nodes. $A$ free link symbol is a product of free knot symbols, no two of 
which have a node in common. Note that each free link symbol can be regarded as an element of the symmetric group $S_{v}$.

(ii) Let $\phi: \Sigma_{A} \rightarrow\{0,1\}$ be defined by $\phi(w)=f(\pi(w))$. Define the trip number $t(\gamma)$ of the free knot symbol $\gamma=\left(i_{1}, i_{2}, \ldots, i_{r}\right)$ by

$$
t(\gamma)=\phi\left(i_{1}\right)+\phi\left(i_{2}\right)+\cdots+\phi\left(i_{r}\right) .
$$

For a free link symbol $\delta=\delta_{1} \delta_{2} \cdots \delta_{p}$, where $\delta_{1}, \ldots, \delta_{p}$ are free knot symbols, define

$$
t(\delta)=\sum_{i=1}^{p} t\left(\delta_{i}\right)
$$

Similarly, let $l(\gamma)$ denote the number of nodes in $\gamma$ and set $s(\gamma)=1$ if $r$ is even and $=0$ if $r$ is odd. Extend this to free link symbols by defining

$$
l(\delta)=\sum_{i=1}^{p} l\left(\delta_{i}\right) \quad \text { and } \quad s(\delta)=\sum_{i=1}^{p} s\left(\delta_{i}\right) .
$$

Example 1. For $\kappa=\left(x^{3} y \overline{0}, y^{3} x \overline{0}\right.$, we obtain the matrix

$$
A=\left(\begin{array}{llllllll}
0 & 1 & 1 & 0 & 0 & 0 & 0 & 0 \\
0 & 0 & 0 & 1 & 0 & 0 & 0 & 0 \\
0 & 0 & 0 & 0 & 1 & 1 & 0 & 0 \\
0 & 0 & 0 & 0 & 0 & 0 & 1 & 1 \\
1 & 1 & 0 & 0 & 0 & 0 & 0 & 0 \\
0 & 0 & 1 & 1 & 0 & 0 & 0 & 0 \\
0 & 0 & 0 & 0 & 1 & 0 & 0 & 0 \\
0 & 0 & 0 & 0 & 0 & 1 & 1 & 0
\end{array}\right)
$$

and the free knot symbols are (12475), (1248635), (124875), (135), (136475), (136487), (235), (2475), (248635), (486), and the corresponding free link symbols are all the free knot symbols together with the products $(135)$ (486) and (235) (486).

Let $\mathscr{P}$ denote the set $\left\{\kappa \in K^{0}: \kappa\right.$ is prime $\}$, which is an open subset of $K^{0}$.

Theorem 1. There exists a continuous, strictly positive function $\lambda: \mathscr{P} \rightarrow \mathbb{R}$ such that, for any Lorenz semiflow with kneading invariant $\kappa$,

$$
\#\{\tau: b(\tau) \leqq m\} \sim \frac{e^{\lambda(\kappa)}}{\left(e^{\lambda(\kappa)}-1\right)} \frac{e^{\lambda(\kappa) m}}{m}
$$

as $m \rightarrow \infty$ through the positive integers. When $k_{l}, k_{r} \in Z, u=\lambda(\kappa)$ is the unique positive solution to

$$
\sum_{\gamma} e^{-t(\gamma) u}=1
$$

where the sum is over all free link symbols $\gamma$.

Proof. Let $\kappa \in K^{0}, \quad \kappa=\left(k_{l}, k_{r}\right)$ be prime and first suppose $k_{l}, k_{r} \in Z$. By Proposition 1, there is a Markov linear realisation $T$ of $\kappa$. Define the "braid index zeta function" for the flow $\Phi_{t}$ with Poincare map $T$, by

$$
\zeta_{T}(s)=\prod_{\tau}\left(1-e^{-s b(\tau)}\right)^{-1}
$$

for any $s \in \mathbb{C}$, whenever the infinite product converges. 
Each closed orbit $\tau$ of $\Phi_{t}$ defines a positive braid on $t(\tau)$ strands, which contains a full twist. (See [BW1], Sect. 5 or the proof of Lemma 5). By [FW], Corollary 2.4, if $b \in B_{n}$ is a positive braid and $b=a \Delta^{2}$, where $\Delta^{2}$ is the full twist braid and $a \in B_{n}$ is positive, then $n$ is the braid index of $b$. Thus we conclude that $b(\tau)=t(\tau)$ for all closed orbits $\tau$.

Using the observation that $k t(\tau)=f^{n}(\xi)$, whenever $T^{n} \xi=\xi, n=k p$, and $p$ is the least period of $\xi$, we may rewrite $\zeta_{T}(s)$ as

$$
\zeta_{T}(s)=\exp \sum_{n=1}^{\infty} \frac{1}{n} \sum_{\xi \in \operatorname{Fix}\left(T^{n}\right)} e^{-s f^{n}(\xi)} .
$$

As we observed in Sect. 1, the map $\pi$ is one-one except for at most a countable number of points, and is everywhere at most bounded-to-one, so using standard arguments in symbolic dynamics,

$$
\zeta_{T}(s)=\zeta_{\sigma}(s)
$$

wherever this makes sense. (Here $\zeta_{\sigma}$ is the symbolic analogue of $\zeta_{T}$ as defined in [P2].) By Lemma $4, f^{N} \geqq 1$ for some $N$, so we may now apply the not weak mixing case of the main result in [P2] to deduce the formula in (3.1).

In particular, $\zeta_{\sigma}(s)$ is analytic and non-zero in an open neighbourhood of $\operatorname{Re}(s) \geqq \lambda$ except for a simple pole at $\operatorname{Re}(s)=\lambda$, where $\lambda>0$ is uniquely determined by $P(-\lambda \phi)=0$, by [P2], Proposition 1 . For $u \in \mathbb{R}$ define a $v \times v$ matrix $B_{u}$ by

$$
B_{u}(i, j)=A(i, j) e^{-u \phi(i)},
$$

which is irreducible by Lemma 4 . Then

$$
\operatorname{det}\left(B_{u}-y I\right)=\sum_{\rho \in S_{v}}(-1)^{\operatorname{sign}(\rho)}\left(B_{u}-y I\right)(1, \rho(1)) \cdots\left(B_{u}-y I\right)(v, \rho(v)) .
$$

Let $\gamma$ be a free link symbol. Observe that there are precisely $v-l(\gamma)$ symbols in $\{1,2, \ldots, v\}$ which are not nodes of the free link symbol $\gamma$. For each such symbol $j$ say, $A(j, j)=0$ by virtue of the fact that $c \in S$. (See Sect. 1.)

Write $\gamma=\gamma_{1} \gamma_{2} \ldots \gamma_{r}$ as a product of free knot symbols, where $\gamma_{i}=\left(k_{1}^{(l)}, \ldots, k_{d_{i}}^{(i)}\right)$ and let $k_{1}^{(r+1)}, \ldots, k_{d_{r+1}}^{(r+1)}$ be those symbols in $\{1,2, \ldots, v\}$, which are not nodes of $\gamma$. Then

$$
\left(B_{u}-y I\right)\left(k_{j}^{(l)}, \gamma_{i}\left(k_{j}^{(i)}\right)\right)=e^{-u \phi\left(k_{J}^{(l)}\right)} \quad \text { for } j=1, \ldots, d_{i} \quad \text { and } \quad i=1, \ldots, r
$$

and

$$
\left(B_{u}-y I\right)\left(k_{j}^{(r+1)}, \gamma_{j}\left(k_{j}^{(r+1)}\right)\right)=-y, \quad \text { for } j=1, \ldots, d_{r+1}=v-l(\gamma) .
$$

So we have

$$
\begin{aligned}
\left(B_{u}-y I\right)(1, \gamma(1))\left(B_{u}-y I\right)(2, \gamma(2)) \cdots\left(B_{u}-y I\right)(v, \gamma(v)) \\
=\left(\prod_{i=1}^{r} \prod_{j=1}^{d_{i}}\left(B_{u}-y I\right)\left(k_{j}^{(i)}, \gamma_{i}\left(k_{j}^{(i)}\right)\right)\right) \\
\quad \cdot\left(\left(B_{u}-y I\right)\left(k_{1}^{(r+1)}, \gamma_{1}\left(k_{1}^{(r+1)}\right)\right) \cdots\left(B_{u}-y I\right)\left(k_{d_{r+1}}^{(r+1)}, \gamma_{r}\left(k_{d_{r+1}}^{(r+1)}\right)\right)\right)
\end{aligned}
$$




$$
\begin{aligned}
& =\left(\prod_{i=1}^{r} \prod_{j=1}^{d_{i}} e^{-u \phi\left(k_{j}^{(l)}\right)}\right) \cdot(-y)^{\nu-l(\gamma)}=\left(\prod_{i=1}^{r} e^{-u t\left(\gamma_{l}\right)}\right) \cdot(-y)^{\nu-l(\gamma)} \\
& =e^{-u t(\gamma)}(-y)^{\nu-l(\gamma)} .
\end{aligned}
$$

Substituting into (3.4) gives

$$
\operatorname{det}\left(B_{u}-y I\right)=(-y)^{v}+\sum_{\gamma}(-1)^{s(\gamma)+v-l(\gamma)} e^{-u t(\gamma)} y^{v-l(\gamma)} .
$$

Let $y=y(u)$ denote the largest positive solution to

$$
\operatorname{det}\left(B_{u}-y I\right)=0 .
$$

From [P2], $y(u)=e^{P(-u \phi)}$. Thus $\lambda>0$ is uniquely determined by $y(\lambda)=1$. We remark that $s(\gamma)+l(\gamma) \equiv 1(\bmod 2)$, for all free link symbols $\gamma$. Let $x(u)=y(u)^{-1}$. Then dividing (3.5) by $(-1)^{v} y(u)$, and substituting $x$ gives that

$$
\sum_{\gamma} e^{-t(\gamma) u} x^{l(\gamma)}=1
$$

However, there is always precisely one solution $x=x(u)$ to this equation for each $u>0$. Thus $\lambda$ is determined uniquely by (3.2).

Now suppose that $k_{l}, k_{r} \in K^{0} \backslash(Z \times Z)$. By Lemma 1 (ii), $Z$ is dense in $X$, and hence $(Z \times Z) \cap K^{0}$ is dense in $K^{0}$. So we may choose kneading invariants $a^{(n)}=$ $\left(a_{l}^{(n)}, a_{r}^{(n)}\right), b^{(n)}=\left(b_{l}^{(n)}, b_{r}^{(n)}\right) \in K^{0}$ such that

$$
\begin{aligned}
& \left(a_{l}^{(n)}, a_{r}^{(n)}\right),\left(b_{l}^{(n)}, b_{r}^{(n)}\right) \in(Z \times Z) \cap K^{0}, \\
& a_{l}^{(n)}<k_{l}<b_{l}^{(n)}, \quad b_{r}^{(n)}<k_{r}<a_{r}^{(n)},
\end{aligned}
$$

and

$$
a_{l}^{(n)} \uparrow k_{l}, \quad b_{l}^{(n)} \downarrow k_{l}, \quad a_{r}^{(n)} \downarrow k_{r}, \quad b_{r}^{(n)} \uparrow k_{r} \quad \text { as } n \rightarrow \infty .
$$

Since $\kappa$ is prime, and since the prime kneading invariants form an open subset of $K^{0}$ by Lemma 1(ii), we may assume that $a^{(n)}$ and $b^{(n)}$ are prime, for all $n$.

Choose realisations $U^{(n)}, V^{(n)}$ for $a^{(n)}, b^{(n)}$ respectively. Using relation (2.2), and the observation that the kneading word of a closed orbit determines its braid index, independent of the realisation map, we have

$$
\#\left\{\rho^{(n)}: b\left(\rho^{(n)}\right) \leqq m\right\} \leqq \#\{\tau: b(\tau) \leqq m\} \leqq \#\left\{\gamma^{(n)}: b\left(\gamma^{(n)}\right) \leqq m\right\},
$$

where $\gamma^{(n)}$ (respectively $\rho^{(n)}$ ) denotes a closed orbit of the Lorenz semiflow with Poincaré map $U^{(n)}$ (respectively $V^{(n)}$ ). Let $\lambda^{(n)}$ (respectively $\mu^{(n)}$ ) be the constants given by (3.2). Then $\lambda^{(n)}$ is monotonic decreasing, since by (3.8) and (2.2) we are deleting closed orbits as $n$ increases, and bounded below (by $\mu^{(1)}$ ), so $\lambda^{(n)} \downarrow \lambda_{+}$say. Similarly, $\mu^{(n)} \uparrow \lambda_{-}$. Now suppose that $\lambda_{+} \neq \lambda_{-}$. Then

$$
\begin{aligned}
& \frac{1}{m} \lim _{m \rightarrow \infty} \log \left(\#\left\{\gamma^{(n)}: b\left(\gamma^{(n)}\right) \leqq m\right\}-\#\left\{\rho^{(n)}: b\left(\rho^{(n)}\right) \leqq m\right\}\right) \\
& \quad=\lambda^{(n)}-\mu^{(n)} \geqq \lambda_{+}-\lambda_{-}>0
\end{aligned}
$$


for all $n \geqq 1$. Thus there is a closed orbit $\tau$ (in fact infinitely many) such that the kneading word $w(\tau) \in X_{1}$ of $\tau$ is allowable with respect to the $a^{(n)}$ for all $n$, but not allowable with respect to $b^{(n)}$ for all $n$, (in the sense of inequality (2.2)).

From (2.2), given $n \geqq 1$, there exists $q$ such that either $\sigma^{q} w(\tau)<b_{l}^{(n)}$ or $b_{r}^{(n)}<\sigma^{q} w(\tau)$. At least one of these two inequalities holds for infinitely many values of $n$, so say it is $\sigma^{q} w(\tau)<b_{l}^{(n)}$. Since $w(\tau)$ is periodic word in $X_{1}$, we may let $N$ be the least natural number such that $\sigma^{N} w(\tau)=w(\tau)$. Thus we can always choose $q$ from the set $\{0,1, \ldots, N-1\}$. So for some fixed $q_{0} \in\{0,1, \ldots, N-1\}$, we can find an infinite sequence $\left(n_{j}\right)$ such that $\sigma^{q_{0}} w(\tau)<b_{l}^{\left(n_{j}\right)}$, for $j=1,2, \ldots$. Since $b_{l}^{\left(n_{j}\right)} \rightarrow k_{l}$ as $n \rightarrow \infty$, we have $\sigma^{q_{0}} w(\tau) \leqq k_{l}$. Further, since $k_{l}$ is not eventually periodic, $\sigma^{q_{0}} w(\tau)<k_{l}$. Since $a_{l}^{(n)} \rightarrow k_{l}$, we can find $n_{0}$ such that $\sigma^{q_{0}} w(\tau)<a_{l}^{\left(n_{0}\right)}$, which by (2.2) shows that $w(\tau)$ is not allowable with respect to $a^{\left(n_{0}\right)}$, giving a contradiction. Thus $\lambda_{+}=\lambda_{-}=\lambda$, and $\lambda>0$ since $\lambda>\mu^{(1)}$.

The above argument can easily be adapted to cover the cases $\left(k_{l}, k_{r}\right) \in$ $K^{0} \cap\left(Z^{c} \times Z\right)$ and $\left(k_{l}, k_{r}\right) \in K^{0} \cap\left(Z \times Z^{c}\right)$. In both these cases, one of the inequalities in (3.9) will be an equality.

We now briefly indicate how to extend formula (3.1) to kneading invariants $\kappa \in K^{0} \backslash(Z \times Z)$. Since these ideas are fairly well known, we shall only give a brief outline. It is convenient to rescale our zeta function as

$$
\zeta(s)=\exp \sum_{n=1}^{\infty} \frac{1}{n} \sum_{\operatorname{Fix}\left(T^{n}\right)} e^{-s \lambda f^{n}(x)}=\prod_{\tau}\left(1-e^{-s \lambda b(\tau)}\right)^{-1},
$$

where $\lambda=\lambda(\kappa), T$ is the linear realisation of $\kappa$ (which is given by Proposition 1), and $f: I \rightarrow\{0,1\}$. We make the following observations:

(i) $\zeta(s)$ is analytic and non-zero for $\operatorname{Re}(s)>1$.

(ii) $\zeta(s)$ is meromorphic for $\operatorname{Re}(s)>0$.

(iii) $\zeta(s)$ is analytic and non-zero on $\operatorname{Re}(s)=1$ except for simple poles at $s=1+\frac{2 \pi i k}{\lambda}$, for each $k \in \mathbb{Z}$.

Formula (3.1) then follows using the analogy with the Prime Number Theorem in $[\mathrm{P} 2]$.

To prove (i), it suffices to note that, by the continuity of $\lambda$,

$$
\limsup _{n \rightarrow \infty}\left|\sum_{x \in \operatorname{Fix}\left(T^{n}\right)} e^{-s \lambda f^{n}(x)}\right|^{1 / n} \leqq \limsup _{n \rightarrow \infty}\left(\sum_{x \in \operatorname{Fix}\left(T^{n}\right)} e^{-\operatorname{Re}(s) \lambda f^{n}(x)}\right)^{1 / n}<1
$$

for $\operatorname{Re}(s)>1$.

To prove (ii), note that $f$ is a function of bounded variation, so we can apply Theorem 2(i) in $[\mathrm{BK}]$ to $\zeta(s)$.

To prove (iii), note that $s=1+i$ is a pole of order $\mathrm{p}$ of $\zeta(s)$ if and only if 1 is an eigenvalue of the Ruelle Perron Frobenius operator, $\mathscr{L}_{(-1+i t) \lambda f}$, of multiplicity $\mathrm{p}$, by applying [BK], Theorem 2(ii). Using the convexity argument of [PP, p. 40], $\mathscr{L}_{-(1+\mathrm{it}) \lambda f}$ has 1 as an eigenvalue if and only if $s=1+\frac{2 \pi i k}{\lambda}$ and further, the eigenvalue 1 is necessarily simple.

Remark 2. (i) By carrying out some simple manipulations involving the zeta function $\zeta(s)$, we can replace Eq. (3.1) by

$$
\#\{\tau: b(\tau)=m\} \sim \frac{e^{\lambda m}}{m} \quad \text { as } m \rightarrow \infty .
$$


This shows, in particular, that all large numbers are braid indices for closed orbits.

(ii) Let

$$
\mathscr{Q}=\left\{(\beta, \alpha) \subset \mathbb{R}^{2}: T_{\beta, \alpha} \text { is locally onto with } T(0) \neq 0, T(1) \neq 1\right\}
$$

which is an open subset of $\mathbb{R}^{2}$, calculated explicitly in [G]. The map $\mathscr{Q} \rightarrow \mathscr{P}$ given by $(\beta, \alpha) \mapsto \kappa_{T_{\beta, \alpha}}$ is easily seen to be continuous. Thus we may define a continuous map by the composition $\mathscr{Q} \rightarrow \mathscr{P} \rightarrow \mathbb{R}^{+}$which we again denote by $\lambda$.

For a closed orbit $\tau$, let $e_{\min }(\tau)$ and $e_{\max }(\tau)$ denote the minimal and maximal exponents of $x$ in the two variable generalisation of the Jones polynomial $j_{\tau}(x, y)$ of $\tau$, as defined in [FW]. Let

$$
e_{\mathrm{diff}}(\tau)=e_{\max }(\tau)-e_{\min }(\tau)
$$

Corollary 1. Under the hypotheses of Theorem 1, either $\#\left\{\tau: e_{\mathrm{diff}}(\tau) \leqq m\right\}$ is infinite for some $m \geqq 0$, or

$$
\#\left\{\tau: e_{\text {diff }}(\tau) \leqq m\right\} \gg \frac{2 e^{\lambda}}{\left(e^{\lambda}-1\right)} \frac{e^{m \lambda / 2}}{m} \quad \text { as } m \rightarrow \infty .
$$

Proof. By [FW], Corollary $1.10, b(\tau) \geqq(1 / 2) e_{\text {diff }}(\tau)+1$, from which it follows that

$$
\{\tau: b(\tau) \leqq m\} \subseteq\left\{\tau: e_{\mathrm{diff}}(\tau) \leqq 2(m-1)\right\}
$$

The result follows by applying Theorem 1 .

For a kneading invariant $\kappa=\left(k_{l}, k_{r}\right) \in K^{0}$, define non-negative integers $q_{l}, q_{r}$ by $q_{l}=\min \left\{n:\left(\sigma^{n} k_{l}\right)_{0} \neq x\right\}$, and $q_{r}=\min \left\{n:\left(\sigma^{n} k_{r}\right)_{0} \neq y\right\}$. Further, let $d=d(\kappa)$ be defined by $d=q_{l}+q_{r}$. Note that for a $\beta$-transformation $T=T_{\beta, \alpha}$,

$$
q_{l}=\left[\frac{\log (\beta+\alpha-1)-\log \left(\alpha \beta^{2}\right)}{\log (\beta)}\right]+1
$$

and

$$
q_{r}=\left[\frac{\log (2+\alpha \beta-\beta-2 \alpha)-\log (2-\alpha-\beta)}{\log (\beta)}\right]+1 .
$$

For certain $\beta$-transformations $T_{\beta, \alpha}$, we now give bounds on $\lambda$ in Theorem 1 in terms of the parameters $\beta, \alpha$.

Proposition 2. Let $T=T_{\beta, \alpha}$ be a locally onto $\beta$-transformation such that $T(0) \neq 0$ and $T(1) \neq 1$. Let $\lambda$ be given by Theorem 1 . Then

$$
\frac{\beta^{2} \log \beta}{\beta+\alpha-1} \leqq \lambda \leqq d \log \beta
$$

where $d=q_{l}+q_{r}$ is computed from (3.10) and (3.11).

Proof. By Remark 2(ii), it suffices to prove the bounds on $\lambda$ when $T$ is Markov. Since $T(0) \neq 0$ and $T(1) \neq 1$, we have $\kappa_{T} \in K^{0}$ and $q_{l}, q_{r}$ are well defined. By Abramov's Theorem [A], $\lambda$ can be expressed as

$$
\lambda=\sup _{\mu} \frac{h(\mu)}{\int \phi d \mu}
$$


where the supremum is over all $\sigma$-invariant Borel probabilities $\mu$. Let $m_{0}$ be the measure of maximal entropy for $\sigma$. Then

$$
\lambda>\frac{h\left(m_{0}\right)}{\int \phi d m_{0}}=\frac{\log \beta}{m_{0}\left(\pi^{-1}(J)\right)},
$$

since $T_{\beta, \alpha}$ has topological entropy $\log \beta$. Let $\left(e_{1}, e_{2}, \ldots, e_{k}\right)^{t}$ be the normalised right eigen-vector of $A$, as in the proof of Proposition 1. Then, for each cylinder set $[i]=\left\{x \in \Sigma_{A}: x_{0}=i\right\}$, we have $m_{0}([i])=e_{i}$ from the construction of $m_{0}$ in [P4]. Therefore,

$$
m_{0}\left(\pi^{-1}(J)\right)=\sum_{i=r}^{s-1} e_{i}
$$

where $s=\min \{j: A(j, 1)=1\}$, and $r=\min \{j: A(j, s)=1\}$. Thus, we have that $m_{0}\left(\pi^{-1}(J)\right)=|J|$, and hence

$$
\lambda>\frac{\log \beta}{|J|}=\frac{\beta^{2} \log \beta}{\beta+\alpha-1} .
$$

We now prove the upper bound. Note that $\phi^{d} \geqq 1$, (cf. Lemma 4 ), where $d$ is computed from (3.10) and (3.11). Hence we have

$$
\lambda=\sup _{\mu} \frac{h(\mu)}{(1 / d) \int \phi^{d} d \mu} \leqq d \sup _{\mu} h(\mu)=d \log \beta .
$$

\section{Estimates for the Genus of Lorenz Knots}

We first consider a $\beta$-transformation $T=T_{\beta, \alpha}$ with kneading invariant $\kappa_{T}=\left(k_{l}, k_{r}\right) \in$ $K^{0}$. The following lemma extends Corollary 5.3 in [BW1].

Lemma 5. $g(\tau) \geqq \frac{1}{2} t(\tau)(t(\tau)-1)$ for all closed orbits $\tau$. Moreover, there is a closed orbit for which equality holds.

Proof. We use the "positive braid representation" for the Lorenz attractor $H_{L}$, given in [BW1], and illustrated in Fig. 4.2. (Figure 4.1 is an intermediate stage in obtaining Fig. 4.2 from Fig. 1.1).

We remark that any closed orbit with trip number $t$ has a representation as an element of $B_{t}$, and further, this representation is as a positive braid.

Let $\gamma$ denote a closed orbit with kneading word

$$
w(\gamma)= \begin{cases}\overline{x(x y)^{t}} & \text { if } q_{l} \geqq q_{r} \\ \overline{(x y)^{t} y} & \text { if } q_{l}<q_{r}\end{cases}
$$

with trip number $t(\gamma)=t$.

First note that $w(\gamma)$ is allowable. Suppose first that $q_{l} \geqq q_{r}$. Observe that since $T^{\prime}>1$, either $q_{l}>1$ or $q_{r}>1$. Thus we may assume $q_{l}>1$. If the word $w(\gamma)$ is not allowable then

$$
\overline{x(x y)^{t}}<k_{l}
$$

by (1.2). Thus $T(0)<c$ and $T^{2}(0)>c$, and so

$$
\alpha<\frac{1-\alpha}{\beta} \text { and } \alpha+\alpha \beta>\frac{1-\alpha}{\beta},
$$




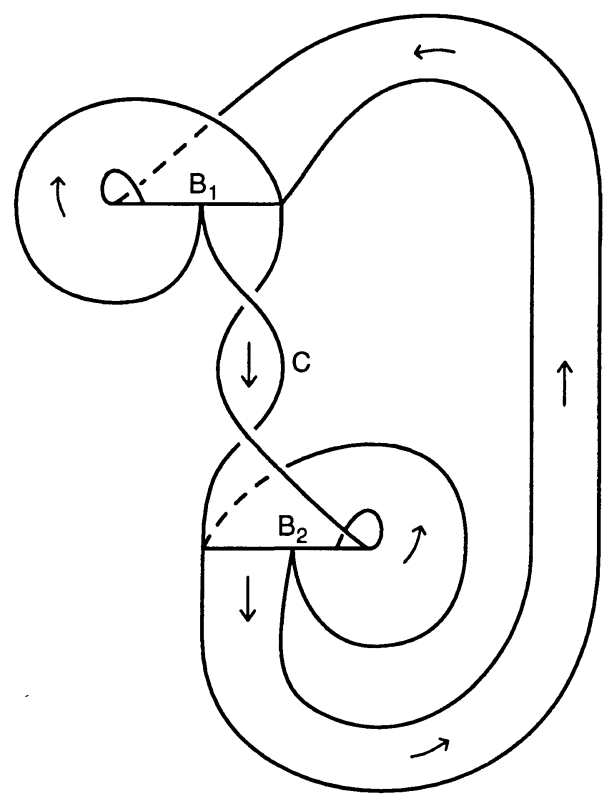

Fig. 4.1.

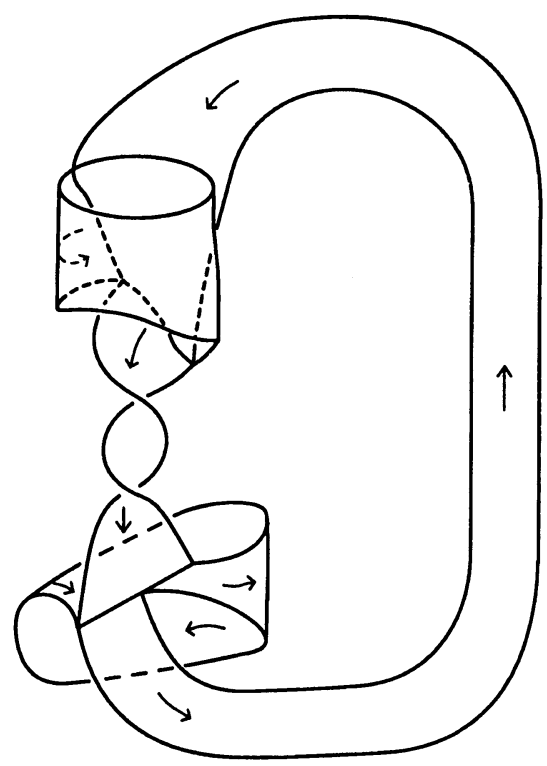

Fig. 4.2.

from which we deduce that $\alpha \beta^{2}>1$. Using the relation $\alpha+\beta \leqq 2$, we obtain

$$
-\beta^{3}+2 \beta^{2}-1>0 \text {. }
$$

However, this is impossible if $\beta>1$, giving a contradiction. If $q_{l}<q_{r}$, we can prove that $w(\gamma)$ is allowable in a similar way.

Note that $\gamma$ has minimal kneading word length over all closed orbits $\tau$ with $t(\tau)=t$. Further, by increasing the word length (keeping $t(\tau)$ fixed), we can only increase the number of self crossings $c(\tau)$ of $\tau$.

At the branch lines $B_{1}, B_{2}, \gamma$ has $t-1$ crossings, and the full twist $C$ contributes $t(t-1)$ self crossings. Thus, $c(\gamma)=t^{2}-1$. Hence for any closed orbit $\tau$ with $t(\tau)=t$

Using the formula

$$
c(\tau) \geqq t^{2}-1 \text {. }
$$

$$
2 g(\tau)=c(\tau)-s(\tau)+1
$$

for a closed orbit $\tau$, represented as a positive braid on $s(\tau)$ strands (from [BW1], Theorem 5.2),

$$
g(\tau) \geqq(1 / 2)\left(t(\tau)^{2}-1-t(\tau)+1\right)=\frac{t(\tau)(t(\tau)-1)}{2}
$$

for any closed orbit $\tau$. Equality holds for the closed orbit $\gamma$.

We now prove an inequality in the opposite direction. Unlike Lemma 5, we cannot always conclude that equality holds for some closed orbit.

\section{Lemma 6.}

$$
g(\tau) \leqq(1 / 2)\left(q_{l}+q_{r}\right) t(\tau)^{2}-t(\tau)+1 / 2
$$

for any closed orbit $\tau$. 
Proof. Let $\gamma$ denote the closed orbit with kneading word

$$
w(\gamma)= \begin{cases}\overline{x^{q_{l}} y^{q_{r}-1}\left(x^{q_{l}} y^{q_{r}}\right)^{t-1}} & \text { if } q_{r} \geqq q_{l} \\ \overline{\left(x^{q_{l}} y^{q_{r}}\right)^{t-1} x^{q_{l}-1} y^{q_{r}}} & \text { if } q_{r}<q_{l}\end{cases}
$$

of trip number $t(\gamma)=t$, (since $q_{l}>1$ or $q_{r}>1$ ).

This time, $w(\gamma)$ may not be allowable as the kneading word of an orbit of $T$. However, it is realisable, for example, as a kneading word of the map $\xi \mapsto 2 \xi$ $(\bmod 1)$. Further, we can always estimate $c(\gamma)$ directly from $w(\gamma)$, without relying on a particular realisation of $w(\gamma)$.

Note that any orbit $\tau$ with $t(\tau)=t$ with word length greater than that of $w(\gamma)$ is definitely not allowable by (2.2). As in Lemma 5, decreasing the word length of $w(\tau)$ can only decrease $c(\tau)$, (keeping $t(\tau)$ fixed). Thus for any closed orbit $\tau$, we have

$$
\begin{aligned}
c(\tau) & \leqq \begin{cases}t(\tau)(t(\tau)-1)+q_{l} t(\tau)^{2}+\left(q_{r}-1\right) t(\tau)^{2} & \text { if } q_{r} \geqq q_{l} \\
t(\tau)(t(\tau)-1)+\left(q_{l}-1\right) t(\tau)^{2}+q_{r} t(\tau)^{2} & \text { if } q_{r}<q_{l}\end{cases} \\
& =t(\tau)^{2}\left(q_{l}+q_{r}\right)-t(\tau),
\end{aligned}
$$

and hence by (4.1),

$$
\begin{aligned}
g(\tau) & \leqq(1 / 2)\left(t(\tau)^{2}\left(q_{l}+q_{r}\right)-t(\tau)-t(\tau)+1\right) \\
& =(1 / 2) t(\tau)^{2}\left(q_{l}+q_{r}\right)-t(\tau)+1 / 2 .
\end{aligned}
$$

Our main result for the genus of Lorenz knots is

Theorem 2. Let $\kappa=\left(k_{l}, k_{r}\right) \in K^{0}$ be prime. For any Lorenz semiflow with kneading invariant $\kappa$, there exists a constant $\lambda>0$ such that

$$
\frac{e^{\lambda(1+1 / d)}}{\left(e^{\lambda}-1\right)} \sqrt{\frac{d}{2}} \frac{e^{\lambda \sqrt{\frac{2}{d}} \sqrt{m}}}{\sqrt{m}} \ll \#\{\tau: g(\tau) \leqq m\} \ll \frac{e^{3 \lambda / 2}}{\sqrt{2}\left(e^{\lambda}-1\right)} \frac{e^{\lambda \sqrt{2} \sqrt{m}}}{\sqrt{m}}
$$

as $m \rightarrow \infty$.

Proof. Let $\kappa=\left(k_{l}, k_{r}\right) \in K^{0}$ be prime. Note that $d \geqq 3$, and hence $q_{l}>1$ or $q_{r}>1$.

The kneading sequences determined by $\kappa$ define the genus of all closed orbits, independent of the realisation map $T$. So we may choose $T$ to be a $\beta$-transformation $T=T_{\beta, \alpha}$ by Proposition 1 .

We consider the right-hand inequality in (4.2) first. If $g(\tau) \leqq m$, then by Lemma $5, t(\tau)^{2}-t(\tau) \leqq 2 m$, and hence

$$
t(\tau) \leqq(1 / 2)(1+\sqrt{1+8 m}) .
$$

Hence by Theorem 1,

$$
\#\left\{\tau: \frac{t(\tau)(t(\tau)-1)}{2} \leqq m\right\} \sim \frac{e^{3 \lambda / 2}}{\sqrt{2}\left(e^{\lambda}-1\right)} \frac{e^{\lambda \sqrt{2} \sqrt{m}}}{\sqrt{m}}
$$


as $m \rightarrow \infty$. Since

$$
\{\tau: g(\tau) \leqq m\} \subseteq\left\{\tau: \frac{t(\tau)(t(\tau)-1)}{2} \leqq m\right\},
$$

we have

$$
\#\{\tau: g(\tau) \leqq m\} \ll \frac{e^{3 \lambda / 2}}{\sqrt{2}\left(e^{\lambda}-1\right)} \frac{e^{\lambda \sqrt{2} \sqrt{m}}}{\sqrt{m}} .
$$

Similarly, for the left-hand inequality in (4.2), we have by Lemma 6 ,

$$
\begin{aligned}
& \#\{\tau: g(\tau) \leqq m\} \geqq \#\left\{\tau:(1 / 2)\left(q_{l}+q_{r}\right) t(\tau)^{2}-t(\tau)+1 / 2 \leqq m\right\} \\
& =\#\left\{\tau: t(\tau) \leqq \frac{1+\sqrt{1+d(2 m-1)}}{d}\right\} \sim \frac{e^{\lambda(1+1 / d)}}{\left(e^{\lambda}-1\right)} \sqrt{\frac{d}{2}} \frac{e^{\lambda \sqrt{2 / d} \sqrt{m}}}{\sqrt{m}}
\end{aligned}
$$

as $m \rightarrow \infty$ by Theorem 1 again. This proves the right-hand inequality in (4.2).

For a knot $K$ in $S^{3}$, let $\Delta(K) \in \mathbb{Z}\left[t, t^{-1}\right]$ denote the Alexander polynomial of $K$. We normalise $\Delta(K)$ so it is a polynomial in $t$ and so that the coefficient of $t^{0}$ is positive. Let deg $\Delta(K)$ denote the degree of $\Delta(K)$.

Corollary 2. Under the hypotheses of Theorem 2, either $\#\{\tau: \operatorname{deg} \Delta(\tau) \leqq m\}$ is infinite for some $m \geqq 0$, or there exists a constant $\lambda>0$ such that

$$
\#\{\tau: \operatorname{deg} \Delta(\tau) \leqq m\} \gg \frac{e^{\lambda(1+1 / d)}}{\left(e^{\lambda}-1\right)} \sqrt{d} \frac{e^{\lambda \sqrt{m / d}}}{\sqrt{m}}
$$

as $m \rightarrow \infty$.

Proof. We use the inequality

$$
\operatorname{deg} \Delta(\tau) \leqq 2 g(\tau)
$$

from [Ro, p. 208], from which it follows that

$$
\{\tau: g(\tau) \leqq m\} \subseteq\{\tau: \operatorname{deg} \Delta(\tau) \leqq 2 m\} .
$$

The result follows by applying Theorem 2 .

Suppose now that $\kappa \notin K^{0}$. First assume that $k_{l}=\bar{x}$. For each $m \geqq 0$ and $n \geqq 1$, let $\gamma_{m, n}$ be the closed orbit with kneading word

$$
w\left(\gamma_{m, n}\right)=\overline{x^{n} y(x y)^{m}} .
$$

A straightforward calculation shows that $t\left(\gamma_{m, n}\right)=m+1$ and $c\left(\gamma_{m, n}\right)=m(m+2)$ for each $n \geqq 1$. Hence, by $(4.1), g\left(\gamma_{m, n}\right)=(1 / 2) m(m+1)$, for each $n \geqq 1$. Thus, it follows that $\#\{\tau: b(\tau)=m\}$ and $\#\{\tau: g(\tau)=(1 / 2) m(m+1)\}$ are infinite for all $m \geqq 0$.

Similarly, we obtain the same result when $k_{r}=\bar{y}$ by considering the closed orbits with kneading words

$$
w\left(\gamma_{m, n}\right)=\overline{(x y)^{m} x y^{n}} .
$$

We summarise these results in the following proposition. 
Proposition 3. If $\kappa \in K \backslash K^{0}$, then

(i) $\#\{\tau: b(\tau)=m\}$ is infinite for each $m \geqq 0$,

(ii) $\sharp\{\tau: g(\tau)=(1 / 2) m(m+1)\}$ is infinite for each $m \geqq 0$.

\section{Average Genus of Lorenz Knots}

For an arbitrary Lorenz semiflow $\Phi_{t}$, and each $\xi \in I \backslash\{c\}$, define the orbit segment $O(\xi)$ by

$$
O(\xi)=\left\{\Phi_{t}(\xi) \in H_{L}: 0 \leqq t<\rho(\xi)\right\} .
$$

Define the function $G: I \times I \rightarrow\{0,1\}$ by

$$
G(\xi, \eta)=\left\{\begin{array}{ll}
1 & \text { if } O(\xi) \text { crosses over } O(\eta) \\
0 & \text { otherwise }
\end{array},\right.
$$

where crossings are counted with respect to the positive braid representation of $H_{L}$. When the Poincare map $T$ is Markov, let $F: \Sigma_{A} \times \Sigma_{A} \rightarrow\{0,1\}$ be given by

$$
F(u, v)=G(\pi(u), \pi(v)) .
$$

Further, let $\omega=m_{-\lambda \phi}$, and let $\pi^{*} \omega$ be the projection of $\omega$ to a measure on $I$ under $\pi$.

There is a natural one-to-one correspondence between the closed orbits of $\Phi_{t}$ and those of $T$. Also, if $T$ is Markov, there is a one-to-one correspondence between closed orbits of $T$ and those of $\sigma: \Sigma_{A} \rightarrow \Sigma_{A}$, (with possibly finitely many orbits excepted).

Let $T$ be Markov, and let $\tau, \gamma$ be generic closed orbits of $\Phi_{t}, \sigma$ respectively, under these correspondences. Define $b(\gamma)=b(\tau), g(\gamma)=g(\tau)$ and $c(\gamma)=c(\tau)$. Let $a: \Sigma_{A} \rightarrow \mathbb{R}$ and let $\Lambda_{a}(\gamma)=a^{n}(x)$, where $x \in \gamma$ and $\sigma^{n} x=x$ and $n>1$ least. Also, for $H: \Sigma_{A} \times \Sigma_{A} \rightarrow \mathbb{R}$, let $\Lambda_{H}(\gamma)=H^{n, n}(x, x)$, where we define

$$
H^{m, n}(x, y)=\sum_{i=0}^{m-1} \sum_{j=0}^{n-1} H\left(\sigma^{i} x, \sigma^{j} y\right) .
$$

The proof of the following lemma uses ideas from the detailed study of dynamical zeta functions made in [P5]. (The techniques are also similar to those in $[\mathrm{Wa}]$.)

Lemma 7. For real valued functions $a_{1}, a_{2} \in C\left(\Sigma_{A}\right)$,

$$
\sum_{b(\gamma) \leqq m} \Lambda_{a_{1}}(\gamma) \Lambda_{a_{2}}(\gamma) \sim \frac{1}{\left(e^{\lambda}-1\right)} \frac{\int a_{1} d \omega \int a_{2} d \omega}{\left(\int \phi d \omega\right)^{2}} m e^{\lambda m}
$$

as $m \rightarrow \infty$, where $\lambda$ is uniquely determined by $P(-\lambda \phi)=0$.

Proof. First let $a_{1}, a_{2}$ be Hölder continuous. We consider the three variable zeta function

$$
\zeta\left(s, z_{1}, z_{2}\right)=\exp \sum_{n=1}^{\infty} \frac{1}{n} \sum_{x \in \operatorname{Fix}\left(\sigma^{n}\right)} e^{-s \lambda \phi^{n}(x)+z_{1} a_{1}^{n}(x)+z_{2} a_{2}^{n}(x)}
$$


which is analytic and non-zero for $\operatorname{Re}(s)>1$ provided that $\left|z_{1}\right|,\left|z_{2}\right|$ are small (depending on $s)$. Note that $\zeta\left(s, z_{1}, z_{2}\right)$ may be rewritten as

Let

$$
\zeta\left(s, z_{1}, z_{2}\right)=\exp \sum_{\gamma} \sum_{k=1}^{\infty} \frac{1}{k} e^{-s k \lambda b(\gamma)+z_{1} k \Lambda_{a_{1}}(\gamma)+z_{2} k \Lambda_{a_{2}}(\gamma)} .
$$

$$
\eta(s)=\left.\frac{\partial}{\partial z_{1}} \frac{\partial}{\partial z_{2}} \log \zeta\left(s, z_{1}, z_{2}\right)\right|_{z_{1}=0=z_{2}}=\sum_{\gamma} \sum_{k=1}^{\infty} k \Lambda_{a_{1}}(\gamma) \Lambda_{a_{2}}(\gamma) e^{-s k \lambda b(\gamma)}
$$

wherever this is well defined.

Using the arguments of [P5], $\eta(s)$ is simply periodic with least period $2 \pi i / \lambda$, (i.e. $\eta(s+2 \pi i k / \lambda)=\eta(s)$ for all $k \in \mathbb{Z})$, wherever this is well defined. Further, $\eta(s)$ is analytic in an open neighbourhood of $\operatorname{Re}(s) \geqq 1$ except for simple poles at the points $s=1+\frac{2 \pi i k}{\lambda}$ for each $k \in \mathbb{Z}$, with residue

Thus,

$$
\frac{\int a_{1} d \omega \int a_{2} d \omega}{\left(\int \phi d \omega\right)^{2}}
$$

$$
\begin{aligned}
\eta(s) & =\frac{\int a_{1} d \omega \int a_{2} d \omega}{\left(\int \phi d \omega\right)^{2}} \frac{1}{\left(1-e^{-\lambda(s-1)}\right)^{2}}+\psi_{1}(s) \\
& =\frac{\int a_{1} d \omega \int a_{2} d \omega}{\left(\int \phi d \omega\right)^{2}} \sum_{n=0}^{\infty}(n+1) e^{-\lambda n(s-1)}+\psi_{1}(s),
\end{aligned}
$$

where $\psi_{1}(s)$ is analytic in an open neighbourhood of $\operatorname{Re}(s) \geqq 1$. Using the argument on [P5, p. 132],

$$
\sum_{k=2}^{\infty} \sum_{\gamma} k \Lambda_{a_{1}}(\gamma) \Lambda_{a_{2}}(\gamma) e^{-s k \lambda b(\gamma)}
$$

is analytic in an open neighbourhood of $\operatorname{Re}(s) \geqq 1$.

From (5.1) and (5.2), we therefore have

$$
\sum_{\gamma} \Lambda_{a_{1}}(\gamma) \Lambda_{a_{2}}(\gamma) e^{-s \lambda b(\gamma)}=\frac{\int a_{1} d \omega \int a_{2} d \omega}{\left(\int \phi d \omega\right)^{2}} \sum_{n=0}^{\infty}(n+1) e^{-\lambda n(s-1)}+\psi_{2}(s),
$$

where $\psi_{2}(s)$ is analytic in an open neighbourhood of $\operatorname{Re}(s) \geqq 1$. Hence

$$
\sum_{n=0}^{\infty}\left(\sum_{b(\gamma)=n} \Lambda_{a_{1}}(\gamma) \Lambda_{a_{2}}(\gamma)-\frac{\int a_{1} d \omega \int a_{2} d \omega}{\left(\int \phi d \omega\right)^{2}}(n+1) e^{\lambda n}\right) e^{-s n \lambda}
$$

is analytic in a neighbourhood of $\operatorname{Re}(s) \geqq 1$. Thus

$$
\sum_{b(\gamma)=n} \Lambda_{a_{1}}(\gamma) \Lambda_{a_{2}}(\gamma)-\frac{\int a_{1} d \omega \int a_{2} d \omega}{\left(\int \phi d \omega\right)^{2}}(n+1) e^{\lambda n} \rightarrow 0
$$

exponentially fast as $n \rightarrow \infty$. Consequently,

$$
\sum_{b(\gamma) \leqq m} \Lambda_{a_{1}}(\gamma) \Lambda_{a_{2}}(\gamma) \sim \frac{\int a_{1} d \omega \int a_{2} d \omega}{\left(\int \phi d \omega\right)^{2}} \sum_{n=0}^{m}(n+1) e^{\lambda n}
$$


as $m \rightarrow \infty$. Noting that

$$
\sum_{n=0}^{m}(n+1) e^{\lambda n} \sim \frac{m e^{\lambda m}}{\left(e^{\lambda}-1\right)}
$$

as $m \rightarrow \infty$ proves the lemma when $a_{1}, a_{2}$ are Hölder continuous.

When $a_{1}, a_{2}$ are merely assumed to be continuous, we approximate $a_{1}, a_{2}$ by Hölder continuous functions.

The following lemma is elementary and can be proved by induction.

Lemma 8. If $H_{j, i}: \mathbb{N} \rightarrow \mathbb{R}^{+}$for $j=1,2$, and $i=1,2, \ldots, N$ are continuous and bounded away from zero and $H_{1, i}(m) \sim H_{2, i}(m)$ as $m \rightarrow \infty$, for each $i$, then

as $m \rightarrow \infty$.

$$
\sum_{i=1}^{N} H_{1, i}(m) \sim \sum_{i=1}^{N} H_{2, i}(m)
$$

Theorem 3. Let $\kappa=\left(k_{l}, k_{r}\right) \in K^{0} \cap(Z \times Z)$ be prime. For any Lorenz semiflow with kneading invariant $\kappa$, there exist constants $\lambda>0, C>0$ such that

$$
\sum_{b(\tau) \leqq m} g(\tau) \sim C m e^{m \lambda}
$$

as $m \rightarrow \infty$. Explicitly, the constant $C$ is given by

and $\lambda$ is given by (3.2).

$$
C=\frac{1}{2\left(e^{\lambda}-1\right)} \frac{\int G d\left(\pi^{*} \omega \times \pi^{*} \omega\right)}{\left(\int f d\left(\pi^{*} \omega\right)\right)^{2}}
$$

Proof. Let $k_{l}, k_{r} \in(Z \times Z) \cap K^{0}$ be prime. Applying Lemma 8 to the formula in Lemma 7 , we have that for continuous functions $a_{1, i}, a_{2, i}$, where $i=1,2, \ldots, N$,

$$
\sum_{b(\gamma) \leqq m} \sum_{i=1}^{N} \Lambda_{a_{1, i}}(\gamma) \Lambda_{a_{2, i}}(\gamma) \sim \frac{1}{\left(e^{\lambda}-1\right)} \frac{\sum_{i=1}^{N} \int a_{1, l} d \omega \int a_{2, i} d \omega}{\left(\int \phi d \omega\right)^{2}} m e^{m \lambda}
$$

as $m \rightarrow \infty$.

Let $\|\cdot\|_{\infty}$ denote the supremum norm on $C\left(\Sigma_{A} \times \Sigma_{A} ; \mathbb{R}\right)$, and for $a_{1}, a_{2} \in$ $C\left(\Sigma_{A} ; \mathbb{R}\right)$, define $\left(a_{1} \cdot a_{1}\right) \in C\left(\Sigma_{A} \times \Sigma_{A} ; \mathbb{R}\right)$ by $\left(a_{1} \cdot a_{2}\right)(x, y)=a_{1}(x) a_{2}(y)$. Let $H \in C\left(\Sigma_{A} \times \Sigma_{A} ; \mathbb{R}\right)$ be arbitrary. By the Stone-Weierstrass Theorem, given $\varepsilon>0$ there exists a number $N \in \mathbb{N}$ and functions $a_{1, l}, a_{2, i} \in C\left(\Sigma_{A} ; \mathbb{R}\right)$ for $i=1,2, \ldots, N$ such that

$$
\left\|H-\sum_{i=1}^{N}\left(a_{1, i} \cdot a_{2, i}\right)\right\|_{\infty}<\varepsilon .
$$

A straightforward approximation argument using (5.5) and (5.6) shows that

$$
\sum_{b(\gamma) \leqq m} \Lambda_{H}(\gamma) \sim \frac{1}{\left(e^{\lambda}-1\right)} \frac{\int H d(\omega \times \omega)}{\left(\int \phi d \omega\right)^{2}} m e^{\lambda m}
$$

as $m \rightarrow \infty$.

Next, we note that $F$ is the characteristic function of a set $B \subset \Sigma_{A} \times \Sigma_{A}$ with $(\omega \times \omega)(\partial B)=0$. Thus by approximation, we may conclude that (5.7) holds with $F$ replacing $H$. Noting that $c(\gamma)=\Lambda_{F}(\gamma)$, we therefore have

$$
\sum_{b(\gamma) \leqq m} c(\gamma) \sim \frac{1}{\left(e^{\lambda}-1\right)} \frac{\int F d(\omega \times \omega)}{\left(\int \phi d \omega\right)^{2}} m e^{m \lambda}
$$

as $m \rightarrow \infty$. 
Using our correspondence between closed orbits of $\Phi_{t}$ and those of $\sigma$, we may replace $(5.8)$ by

$$
\sum_{b(\tau) \leqq m} c(\tau) \sim \frac{1}{\left(e^{\lambda}-1\right)} \frac{\int F d(\omega \times \omega)}{\left(\int \phi d \omega\right)^{2}} m e^{m \lambda}
$$

as $m \rightarrow \infty$. By Theorem 1 ,

$$
\sum_{b(\tau) \leqq m} b(\tau) \leqq m \sum_{b(\tau) \leqq m} 1 \sim C^{\prime} e^{\lambda m}
$$

as $m \rightarrow \infty$, for a constant $C^{\prime}>0$.

Thus by (4.1),

$$
\begin{aligned}
\frac{1}{m e^{m \lambda}} \sum_{b(\tau) \leqq m} g(\tau) & =\frac{1}{2}\left(\sum_{b(\tau) \leqq m} c(\tau)+\sum_{b(\tau) \leqq m}(1-b(\tau))\right) \\
& \rightarrow \frac{1}{2\left(e^{\lambda}-1\right)} \frac{\int F d(\omega \times \omega)}{\left(\int \phi d \omega\right)^{2}}
\end{aligned}
$$

as $m \rightarrow \infty$. It remains to note that

$$
\frac{\int F d(\omega \times \omega)}{\left(\int \phi d \omega\right)^{2}}=\frac{\int G d\left(\pi^{*} \omega \times \pi^{*} \omega\right)}{\left(\int f d\left(\pi^{*} \omega\right)\right)^{2}} .
$$

Remarks 3(i). For a Lorenz semiflow with first return map a $\beta$-transformation $T_{\beta, \alpha}$, the function $G$ is the characteristic function of the set

$$
\left\{(\xi, \eta) \in I \times I:(1-\alpha(1+\beta)) / \beta^{2} \leqq \xi \leqq(1-\alpha) / \beta,(1-\alpha) / \beta \leqq \eta \leqq \beta \xi+\alpha\right\} .
$$

(ii) Using results on the analytic domain of zeta functions for non-Markov maps of the interval, it should be possible to prove the corresponding version of Theorem 3.

Corollary 3. Let $\kappa=\left(k_{l}, k_{r}\right) \in K^{0} \cap(Z \times Z)$ be prime. For any Lorenz semiflow with kneading invariant $\kappa$, there is a constant $C_{1}>0$ such that

$$
\frac{\sum_{b(\tau) \leqq m} g(\tau)}{\sum_{b(\tau) \leqq m} 1} \sim C_{1} m^{2} \quad \text { as } m \rightarrow \infty .
$$

Proof. Combine Theorem 3 with Theorem 1.

In the spirit of Corollaries 1 and 2, we can combine estimates involving the Jones and Alexander polynomials. For example, we have

Corollary 4. Let $\kappa \in K^{0} \cap(Z \times Z)$ be prime. For any Lorenz semiflow with kneading invariant $\kappa$, there exists a constant $C_{2}>0$ such that

$$
\frac{\sum_{b(\tau) \leqq m} \operatorname{deg} \Delta(\tau)}{\sum_{b(\tau) \leqq m} 1} \ll C_{2} m^{2} \quad \text { as } m \rightarrow \infty \text {. }
$$

\section{Renormalisation of Lorenz Semiflows}

In this section, we discuss an example in [P3], to illustrate the effect of renormalisation of the flow on the link of Lorenz knots. We consider the centrally symmetric 


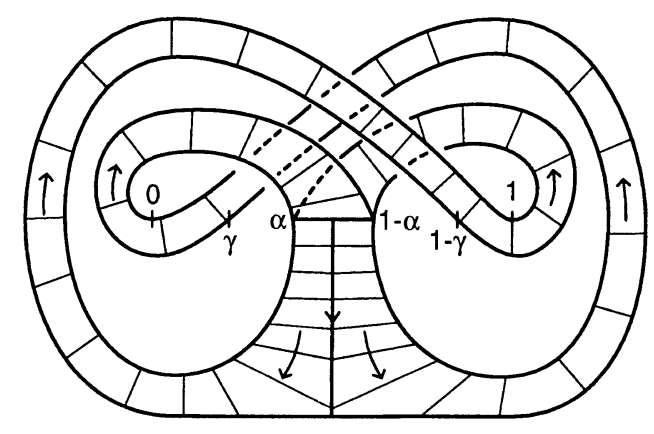

Fig. 6.1.

Poincaré map

$$
T(\xi)=\beta \xi+\left(1-\frac{\beta}{2}\right)(\bmod 1) .
$$

We assume that $T$ is Markov, and that $\beta>\sqrt{2}$, (which ensures that $T$ is locally onto by [W1], Proposition 1). Let $\Phi_{t}: H_{L} \rightarrow H_{L}$ be the Lorenz semiflow with Poincare map $T$, and let $\kappa$ denote the (prime) kneading invariant of $T$.

Next, let $\Psi_{t}$ be the Lorenz semiflow with Poincaré map

$$
U(\xi)=\sqrt{\beta} \xi+\left(1-\frac{\sqrt{\beta}}{2}\right)(\bmod 1),
$$

with kneading invariant $\chi$. It is immediate that $\chi$ is 1 -renormalisable, with renormalisation $\kappa$. Our aim is to compare the links of closed orbits of $\Phi_{t}$ and $\Psi_{t}$.

Let $\alpha=1-(1 / 2) \sqrt{\beta}, \gamma=U(1-\alpha)$ and define intervals, $I_{1}, \ldots, I_{5}$ by

$$
\begin{aligned}
& I_{1}=[0, \gamma], \quad I_{2}=(\gamma, \alpha), \quad I_{3}=[\alpha, 1-\alpha], \\
& I_{4}=(1-\alpha, 1-\gamma), \quad I_{5}=[1-\alpha, 1] .
\end{aligned}
$$

Also let

$$
H_{1}=\left\{\Psi_{t}(\xi): 0 \leqq t<\rho(\xi), \xi \in I_{1} \cup I_{3} \cup I_{5}\right\}
$$

and

$$
H_{2}=\left\{\Psi_{t}(\xi): 0 \leqq t<\rho(x), \xi \in I_{2} \cup I_{4}\right\},
$$

so that $H_{L}=H_{1} \cup H_{2}$. The following lemma is a reformulation of results in [P3].

\section{Lemma 10.}

(i) $\Psi_{t} H_{1}=H_{1}$ for all $t \geqq 0$.

(ii) $U \mid I_{3}$ is disjoint from $I_{3}$, and $U^{2} I_{3}=I_{3}$. Moreover, if $U^{2} \mid I_{3}$ is rescaled to a map $I \rightarrow I$, it is equal to $T$.

(iii) The region $\mathrm{H}_{2}$ contains a single repelling closed orbit which intersects $I$ twice. The remaining orbits of points of $H_{2}$ converge to $H_{1}$ and are captured in this region in a finite amount of time.

The lemma is best illustrated by Fig. 6.1. (The region $H_{1}$ is shaded and the region $\mathrm{H}_{2}$ is unshaded.) 
The interval $I_{3}$ contains symmetric asscociates $(\alpha, 1 / 2),(1 / 2,1-\alpha)$ which are mapped one-to-one onto $(1-\gamma, 1),(0, \gamma)$ respectively. Moreover, $U$ maps each of $(0, \gamma),(1-\gamma, 1)$ one-one into $I_{3}$.

Following the procedure in Sect. 4, we now give a positive braid representation for the template $H_{1}$.

By Lemma 10(ii), we take $T: I \rightarrow I$ to be the Poincare map on the branch line of $H_{1}$. Let $J$ be as in Sect. 3, and let $g: I \rightarrow I$ be the characteristic function of $I \backslash J$, so $g=1-f$, and define $\psi: \Sigma_{A} \rightarrow\{0,1\}$ by $\psi(w)=g(\pi(w))$. For a free knot symbol $\gamma=\left(i_{1}, i_{2}, \ldots, i_{r}\right)$, let $t^{\prime}(\gamma)=\psi\left(i_{1}\right)+\cdots+\psi\left(i_{r}\right)$, and extend this to free knot symbols as in Sect. 3. For a closed orbit $\tau$, with $\tau \cap I=\left\{\xi, T \xi, \ldots, T^{n-1} \xi\right\}$ and $n>1$ least, let $t^{\prime}(\tau)=g^{n}(\xi)$.

Our main observation is that a closed orbit $\tau$ of $\Psi_{t} \mid H_{1}$ with $t^{\prime}(\tau)=t$ can be represented as a positive braid in $B_{t}$, and this braid word contains a full twist. This is evident from Fig. 6.2. We can now prove the following lemma in the same way as Theorem 1.

Lemma 11. Let $u=\lambda_{1}$ denote the unique positive solution to the equation

$$
\sum_{\gamma} e^{-t^{\prime}(\gamma) u}=1
$$

where the sum is over all free knot symbols $\gamma$. Then for the semiflow $\Psi_{t} \mid H_{1}$, we have

$$
\#\{\tau: b(\tau) \leqq m\} \sim \frac{e^{\lambda_{1}}}{\left(e^{\lambda_{1}}-1\right)} \frac{e^{\lambda_{1} m}}{m} \text { as } m \rightarrow \infty .
$$

Proposition 4. Let $\tau^{(0)}, \tau^{(1)}$ denote generic closed orbits of $\Phi_{t}, \Psi_{t}$ respectively, in $H_{L}$. Then there exist constants $\lambda_{0}, \lambda_{1}>0$ given by (3.2), (6.1) respectively,

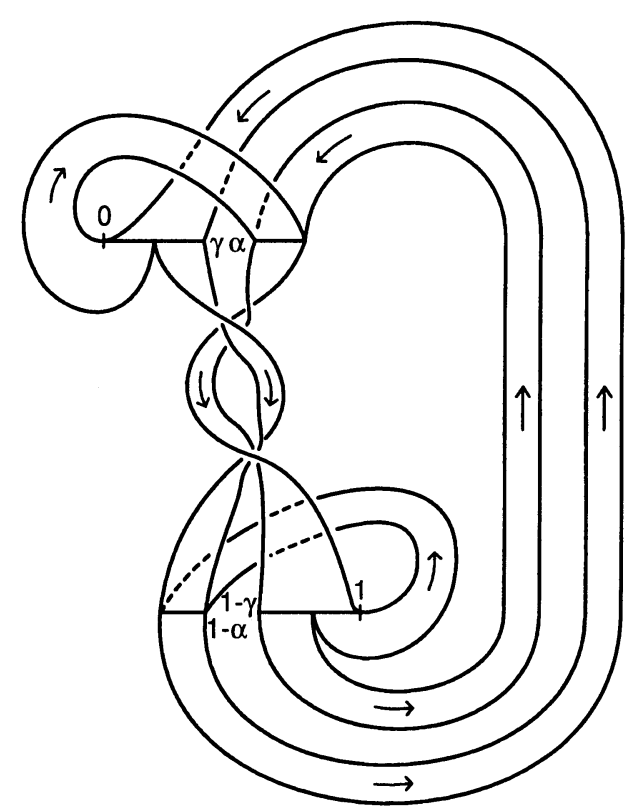

Fig. 6.2. 
such that

$$
\#\left\{\tau^{(i)} ; b\left(\tau^{(i)}\right) \leqq m\right\} \sim \frac{e^{\lambda_{i}}}{\left(e^{\lambda_{l}}-1\right)} \frac{e^{\lambda_{i} m}}{m} \quad \text { as } m \rightarrow \infty, \text { for } i=0,1 .
$$

Further, $\lambda_{1}<\lambda_{0}$, and hence

$$
\frac{\#\left\{\tau^{(1)}: b\left(\tau^{(1)}\right) \leqq m\right\}}{\#\left\{\tau^{(0)}: b\left(\tau^{(0)}\right) \leqq m\right\}} \rightarrow 0
$$

exponentially fast as $m \rightarrow \infty$.

Proof. The case $i=0$ follows from Theorem 1. For the case $i=1$, note that, by Lemma 10, the closed orbits of $\Psi_{t} \mid H_{L}$ and $\Psi_{t} \mid H_{1}$ differ by a single closed orbit. Thus we may apply Lemma 11 to deduce the asymptotic formula given above.

From the lower bound in Proposition $2, \lambda_{0} \geqq 2 \beta \log \beta$. On the other hand, $\psi^{2}=\psi+\psi \circ \sigma>0$, and thus $\lambda_{1} \leqq 2 \log \beta$, as in the proof of the upper bound in Proposition 2. Since $\beta>1$, we have $\lambda_{1}<\lambda_{0}$.

Remark 4. Proposition 4 allows us to conclude that the braid indices of the renormalised semiflow $\Psi$ grow more rapidly than those of $\Phi$.

\section{The Horseshoe Template}

We now apply the techniques developed to analyse Lorenz knots to study the knotted periodic orbits of the Horeshoe template as illustrated in Fig. 7.1.

The template $H_{h}$ may be regarded as a different embedding of the abstract Lorenz template.

We consider Poincaré maps $T: I \rightarrow I$ of the form

(i) $T$ is differentiable for $x \neq c$, for some $c \in(0,1)$,

(ii) $T(\xi) \rightarrow 1$ as $\xi \uparrow c, T(1)=0, T(c)=c$,

(iii) $T^{\prime}(\xi) \geqq \beta$ for all $\xi \in(0, c)$ and $T^{\prime}(\xi) \leqq-\beta$ for all $\xi \in(c, 1)$,

for some $\beta>1$, in which case we write $T \in M_{\beta}$. (For example see Fig. 7.2).

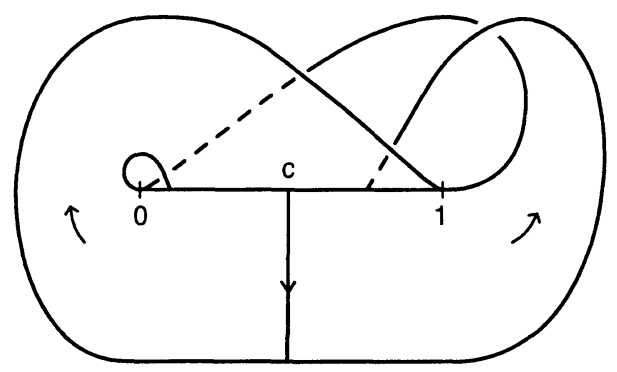

Fig. 7.1.

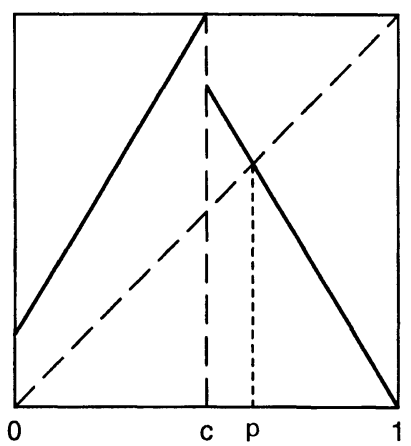

Fig. 7.2. 
In this case a $\beta$-transformation takes the form

$$
T_{\beta, \alpha}(\xi)= \begin{cases}\beta \xi+\alpha & \text { for } 0 \leqq \xi<\beta^{-1}(1-\alpha)=c \\ c & \text { for } \xi=c \\ \beta(1-\xi) & \text { for } c<\xi \leqq 1\end{cases}
$$

where $1<\beta \leqq 2, \alpha \geqq 0$ and $\alpha+\beta \leqq 2$.

Using rule (2.1), each map $T \in M_{\beta}$ determines a space of kneading sequences $Y \subseteq X$. Define a map $\theta: X \rightarrow X$ by writing $\theta(w)=\left(\theta(w)_{j}\right)_{j=0}^{\infty}$, where

$\theta(w)_{j}= \begin{cases}w_{j} & \text { if } w_{j}=x \text { or } y, \text { and the } y \text {-parity of the first } j \text { entries of } w \text { is even } \\ \hat{w}_{j} & \text { if } w_{j}=x \text { or } y, \text { and the } y \text {-parity of the first } j \text { entries of } w \text { is odd } \\ 0 & \text { if } w_{j}=0,\end{cases}$ and

$$
\hat{w}_{j}= \begin{cases}y & \text { if } w_{j}=x \\ x & \text { if } w_{j}=y .\end{cases}
$$

Define an order $<$ on $X$ by writing $w<u$ if $\theta(u) \triangleleft \theta(w)$, where $\triangleleft$ is the usual lexicographical ordering. It is straightforward to check that $<$ is the order on $X$ induced by the natural order on the branch line $I$.

The kneading invariant of $T, \chi=\left(h_{l}, h_{r}\right) \in K$ is defined to be the pair $(k(0)$, $k\left(T\left(c^{+}\right)\right)$. Let $K$ be the space of such pairs, with metric $D$, as in Sect. 2 .

For a sequence $\xi \in I$, a sequence $k(\xi) \in I$ is $T$-admissable if and only if

$$
\begin{aligned}
& \left.\begin{array}{c}
h_{l} \leqq \sigma^{m} k(\xi) \\
\sigma^{m+1} k(\xi) \leqq y h_{l}
\end{array}\right\} \quad \text { if } T^{m}(\xi)<c, \\
& \left.\begin{array}{l}
h_{r} \leqq \sigma^{m} k(\xi) \\
\sigma^{m} k(\xi) \leqq y h_{l}
\end{array}\right\} \quad \text { if } T^{m}(\xi)<c, \\
& \sigma^{m} k(\xi)=0 \text { if } T^{m}(\xi)=c .
\end{aligned}
$$

For a finite, aperiodic word $w$, let $R(w)$ be the number of $y y$ syllables in the word $w$, counted cyclically. For a closed orbit $\tau$ with kneading word $\overline{w(\tau)}$, let $r(\tau)=R(w(\tau))$.

The kneading invariant $\chi$ is called prime if the kneading space $Y$ determined by $\chi$ has the property that, for every non-empty cylinder set $C \subset Y$, there exists $N$ such that $\bigcup_{n=0}^{N} \sigma^{n} C=Y$.

Proposition 5. Let $\chi=\left(h_{l}, h_{r}\right) \in K \cap(Z \times Z)$, and suppose that $\chi$ is prime. Then $T$ is realisable by a Markov $\beta$-transformation $T \in M_{\beta}$.

Proof. As in the proof of Proposition 1, let $L=\left\{\sigma^{n} h_{l}: n \geqq 0\right\}, R=\left\{\sigma^{n} h_{r}\right.$ : $n \geqq 0\}$ and set $E=L \cup R \cup\left\{0 y h_{l}, 0 h_{r}\right\}$, which is a finite set with $\sigma E=E$. Choose any realisation $U$ of $\chi$. Then since $\chi$ is prime, $U$ is locally onto, and hence the transition matrix $A$ is irreducible. Let $e=\left(e_{1}, e_{2}, \ldots, e_{v}\right)$ be the normalised positive eigenvector corresponding to the maximal positive eigenvalue $\lambda$ for $A$. Set $\rho_{0}=0, \rho_{i}=e_{i}+e_{i-1}+\cdots+e_{1}$ for $i=1, \ldots, v$. 
Choose $T$ to be the $\beta$-transformation

$$
T(\xi)=\left\{\begin{array}{ll}
\lambda \xi+\rho_{r} & \text { if } 0 \leqq \xi<\rho_{s} \\
\lambda(1-\xi) & \text { if } \rho_{s}<\xi \leqq 1
\end{array},\right.
$$

where $r=\min \{i: 1 \leqq i \leqq v, A(1, i) \neq 0\}-1$, and $s$ satisfies $k\left(\rho_{s}\right)=0 y h_{l}$.

In view of Proposition 5, we now assume that $T=T_{\beta, \alpha}$ is locally onto.

Lemma 12. For any closed orbit $\tau$,

$$
g(\tau) \geqq(1 / 2)(t(\tau)+r(\tau))^{2}-(7 / 2)(t(\tau)+r(\tau)) .
$$

Moreover, equality holds for some closed orbit $\tau$.

Proof. Let $\rho: I \rightarrow \mathbb{R}^{+}$be the first return time map on $I$. We now give a positive braid representation of the horseshoe template, analogous to that for the Lorenz template. This process comprises two stages.

Let $p$ denote the unique fixed point of $T$, explicitly, $p=\beta^{-1}(2 \beta+\alpha-1)$. Let $\Delta$ denote the closed orbit $\left\{\Phi_{t}(p): 0 \leqq t<\rho(p)\right\}$. Replace $\Delta$ by two parallel copies, (i.e. perform an "orbit splitting" along $\Delta$ as described in the proof of Theorem 2.1 in [BW2]). See Fig. 7.3 for the result of this operation.

Secondly, let $T\left(c^{+}\right)=z^{+}, T\left(c^{-}\right)=z^{-}$, and cut along the orbit segments joining $z^{+}$to $c$ and $z^{-}$to $c$, (Fig. 7.4). Since $z^{+}, z^{-}$do not lie on periodic orbits, this operation leaves the link of periodic orbits invariant.

Rearranging Fig. 7.4 gives Fig. 7.5, and hence Fig. 7.6.

Consider the closed orbit with kneading word $w(\gamma)=\overline{(x y)^{t} y^{r}}$, which satisfies $t(\gamma)=t$, and $r(\gamma)=r$. As in Lemma 5, one can show $w(\gamma)$ is allowable. Also, $c(\gamma)$ minimises $c(\tau)$ over all closed orbits $\tau$ with $t(\tau)=t$, and $r(\tau)=r$. (Any other orbit $\tau$ with the property $t(\tau)=t$ and $r(\tau)=r$ must have greater word length, and hence more self crossings.)

A straightforward calculation gives

$$
c(\gamma) \geqq t(t-1)+(1 / 2) r(r-1)+(1 / 2) t(t-1)+t r+(t-1)(r-1)
$$

by counting crossings at $C_{1}, C_{2}, C_{3}, C_{4}$ and $B_{2}$ respectively,

$$
\geqq(3 / 2) t^{2}+2 t r+(1 / 2) r^{2}-(3 / 2) r-(5 / 2) t .
$$

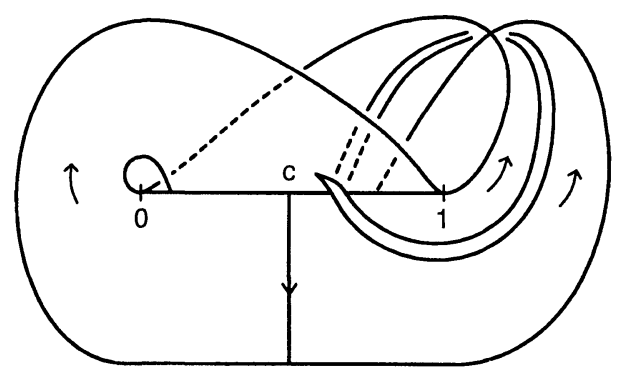

Fig. 7.3. 


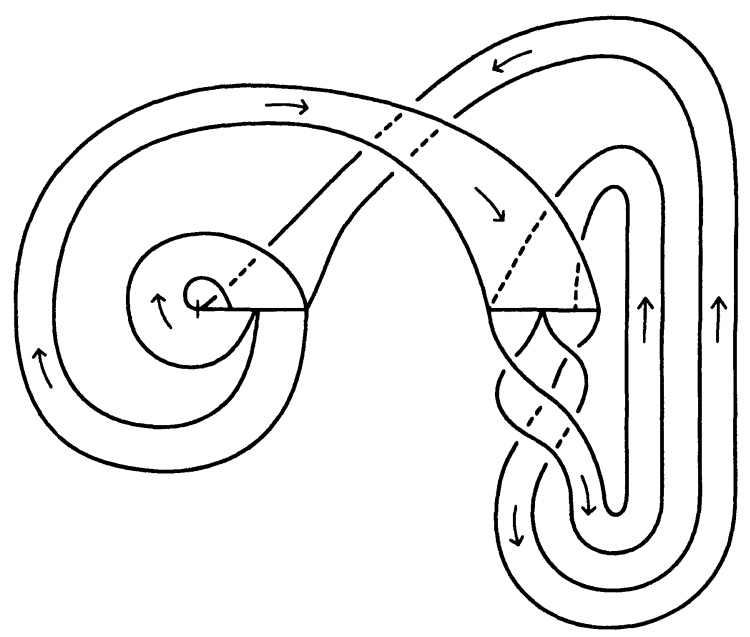

Fig. 7.4.

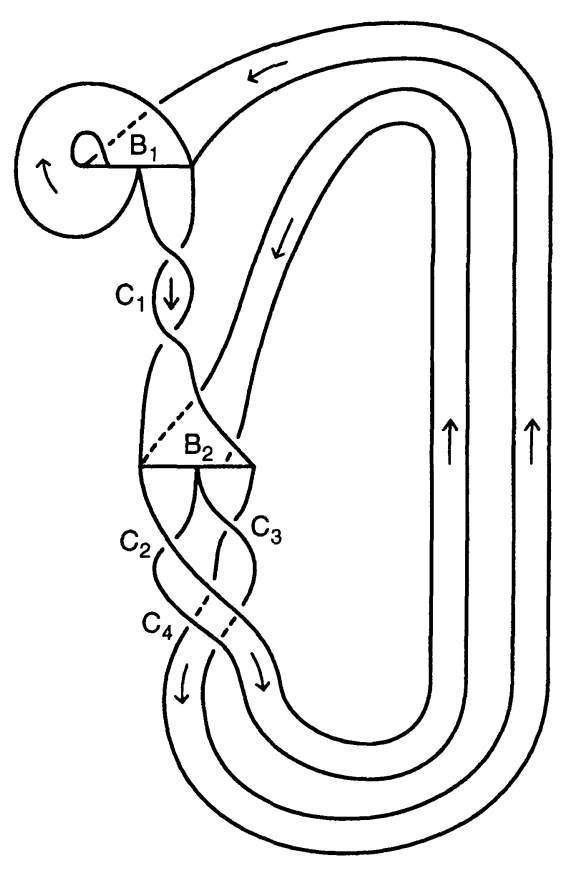

Fig. 7.5.

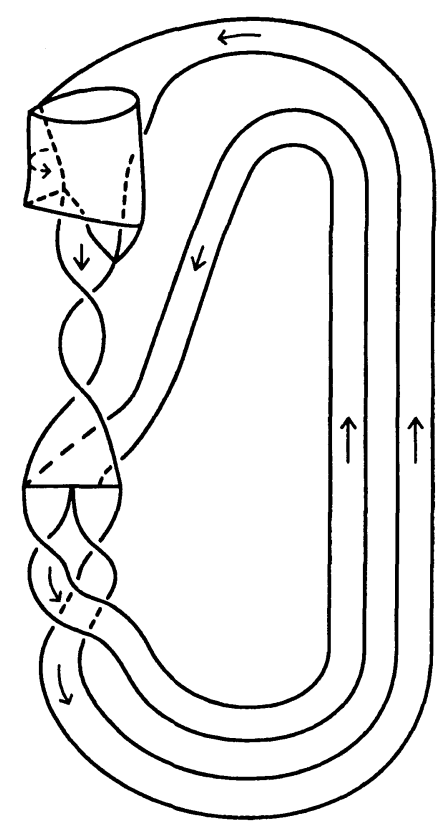

Fig. 7.6.

Thus for any closed orbit $\tau$,

$$
c(\tau) \geqq \frac{3}{2} t(\tau)^{2}+2 t(\tau) r(\tau)+(1 / 2) r(\tau)^{2}-(3 / 2) r(\tau)-(5 / 2) t(\tau) .
$$


Since Fig. 7.6 gives a positive braid representation of each closed orbit $\tau$ of $\Phi_{t}$, on $t(\tau)+r(\tau)$ strands, we have by $(4.1)$,

$$
\begin{aligned}
g(\tau) \geqq & (1 / 2)\left((3 / 2) t(\tau)^{2}+2 t(\tau) r(\tau)+(1 / 2) r(\tau)^{2}\right. \\
& -(3 / 2) r(\tau)-(5 / 2) t(\tau)-t(\tau)-r(\tau)+1) \\
\geqq & (1 / 2)(t(\tau)+r(\tau))^{2}-(7 / 2)(t(\tau)+r(\tau)) .
\end{aligned}
$$

We now prove an inequality in the opposite direction. We assume that $q_{l}>1$, which ensures that $T(0)<c$.

Lemma 13. For any closed orbit $\tau$,

$$
\begin{aligned}
g(\tau) \leqq & \left(t(\tau)^{2} / 4\right)\left(r(\tau)^{2}+3 r(\tau)+2 q_{l}-1\right)-(1 / 4) r(\tau) t(\tau) \\
& -(3 / 2) t(\tau)-(1 / 2) r(\tau)+(1 / 2)
\end{aligned}
$$

Proof. Given $r, t$, set $r^{\prime}=n t$, where $n=[r / t]+1$. Let $\gamma$ denote the closed orbit with kneading word

$$
w(\gamma)=\overline{\left(x^{q_{l}} y^{n+1}\right)^{t-1} x^{q_{l}-1} y^{n+1}}
$$

with $t(\tau)=t$, and $r(\gamma)=r^{\prime} \geqq r$.

If $w(\gamma)$ is not admissable then we can apply the same trick as in Lemma 6. To maximise $c(\gamma)$, we "equidistribute" the $y$ 's amongst the $x$ 's in the kneading word $w(\gamma)$. Then $c(\gamma)$ forms an upper bound for $c(\tau)$ amongst all closed orbits $\tau$ with $t(\tau)=t$ and $r(\tau)=r$.

The full twist $C_{1}$ contributes $t(t-1)$ crossings to $c(\gamma)$, there are at most $\left(q_{l}-1\right) t^{2}$ crossings at $B_{1}$, at most $(1 / 2) r t^{2}$ crossings at $B_{2}$, at most $(1 / 2) r t(r t-1)$ crossings at $C_{2},(1 / 2) t(t-1)$ crossings at $C_{3}$ and at most $t^{2} r$ crossings at $C_{4}$.

Thus

$$
\begin{aligned}
c(\gamma) & \leqq\left(q_{l}-1\right) t^{2}+(1 / 2) r t^{2}+(1 / 2) r t(r t-1)+(1 / 2) t(t-1)+t^{2} r \\
& =t^{2}\left((1 / 2) r^{2}+(3 / 2) r+q_{l}-(1 / 2)\right)-(1 / 2) r t-(1 / 2) t
\end{aligned}
$$

Since a closed orbit with $t(\tau)=t$ and $r(\tau)=r$ is a positive braid on $t+r$ strands, we have by (4.1) that

$$
\begin{aligned}
g(\tau)= & (1 / 2)(c(\tau)-t(\tau)-r(\tau)+1) \\
\leqq & \left(t(\tau)^{2} / 4\right)\left(r(\tau)^{2}+3 r(\tau)+2 q_{l}-1\right)-(1 / 4) r(\tau) t(\tau) \\
& -(3 / 2) t(\tau)-(1 / 2) r(\tau)+(1 / 2)
\end{aligned}
$$

for any closed orbit $\tau$.

Lemma 14. There exists a unique positive real number $\delta$ such that

$$
\#\{\tau: t(\tau)+r(\tau) \leqq m\} \sim \frac{e^{\delta}}{\left(e^{\delta}-1\right)} \frac{e^{\delta m}}{m} \quad \text { as } m \rightarrow \infty .
$$


Proof. We proceed in a similar manner to Lemma 4 and Proposition 1. Define $f: I \rightarrow\{0,1\}$ by $f(\xi)=\mathbf{1}_{I_{1} \cup I_{2}}$, where

$$
I_{1}=\left(\frac{1-\alpha(1+\beta)}{\beta^{2}}, \frac{1-\alpha}{\beta}\right), \quad I_{2}=\left(\frac{1-\alpha}{\beta}, \frac{(1-\alpha)(1+\beta)}{\beta^{2}}\right)
$$

It is not hard to see that $f^{N} \geqq 1$ for $N \geqq q_{l}$. Also, $f^{n}(\xi)=r(\tau)+t(\tau)$ whenever $\xi \in I$ has least period $n$ under $T$. Let $A$ be the irreducible transition matrix given in the proof of Proposition 5. Let $\phi: \Sigma_{A} \rightarrow\{0,1\}$ be defined by $\phi(w)=f(\pi(w))$. Then we may apply the main theorem in [P2] to deduce (7.2), where $\delta>0$ is defined uniquely by $P(-\delta \phi)=0$.

Theorem 4. Let $\chi=\left(h_{l}, h_{r}\right) \in K \cap(Z \times Z)$ be prime and suppose $q_{l}>1$. For any Horseshoe semiflow with kneading invariant $\chi$, there exists a constant $\delta>0$ such that

$$
\frac{e^{\delta}}{\left(e^{\delta}-1\right)} \frac{e^{\delta \sqrt[4]{m}}}{\sqrt[4]{m}} \ll \#\{\tau: g(\tau) \leqq m\} \ll \frac{e^{(9 / 2) \delta}}{\left(e^{\delta}-1\right)} \frac{e^{\delta \sqrt{2 m}}}{\sqrt{m}}
$$

as $m \rightarrow \infty$.

Proof. Using Proposition 5, we can choose the realisation $T$ to be Markov and a $\beta$-transformation, $T=T_{\beta, \alpha}$.

To prove the right-hand side of the inequality in (7.3), note that by Lemma 12 ,

$$
\begin{aligned}
\#\{\tau: g(\tau) \leqq m\} & \leqq \#\left\{\tau:(1 / 2)(t(\tau)+r(\tau))^{2}-(7 / 2)(t(\tau)+r(\tau)) \leqq m\right\} \\
& =\#\left\{\tau: t(\tau)+r(\tau) \leqq \frac{7+\sqrt{49+8 m}}{2}\right\} \sim \frac{e^{(9 / 2) \delta}}{\left(e^{\delta}-1\right) \sqrt{2}} \frac{e^{\delta \sqrt{2 m}}}{\sqrt{m}}
\end{aligned}
$$

as $m \rightarrow \infty$, by Lemma 14 .

To prove the left-hand inequality in (7.3), observe that since $t(\tau)$ and $r(\tau)$ are non-negative, if $t(\tau)+r(\tau) \leqq k$ then $t(\tau) \leqq k$ and $r(\tau) \leqq k$. Hence by Lemma 13,

$$
g(\tau) \leqq(1 / 4) k^{4}+(3 / 4) k^{3}+(1 / 2)\left(q_{l}-1\right) k^{2}-2 k+(1 / 2)
$$

Thus we have that

$$
\begin{aligned}
& \#\{\tau: g(\tau) \leqq m\} \\
& \quad \geqq \#\left\{\tau: t(\tau)+r(\tau) \leqq \sqrt{\frac{-(1 / 2)\left(q_{l}-1\right)+\sqrt{(1 / 4)\left(q_{l}-1\right)^{2}+6+m}}{2}}\right\} \\
& \sim \frac{e^{\delta}}{\left(e^{\delta}-1\right)} \frac{e^{\delta \sqrt[4]{m}}}{\sqrt[4]{m}}
\end{aligned}
$$

as $m \rightarrow \infty$, by Lemma 14 . 


\section{An Estimate on the Genus of Figure of Eight Knots}

This section is a more informal discussion in which we indicate how the results in Sect. 4 for the Lorenz template $H_{L}$ can be applied to analyse the knotted periodic orbits of a more complicated flow. We consider the "figure of eight template" $H_{8}$, which was extensively studied in [BW2], and is illustrated in Fig. 8.1. Let $\Phi_{t}$ denote a semiflow on $H_{8}$, with Poincaré map $T$ along the branch line $I=I_{1} \cup I_{2} \cup I_{3} \cup I_{4}$. (As usual $I=[0,1]$.) A typical example of a Poincaré map $T$ is given in Fig. 8.2. For simplicity, we always assume that $T$ is piecewise linear, and that $\left|T^{\prime}\right|>1$.

Let $\left(a_{i-1}, a_{i}\right)$, for $1 \leqq i \leqq 8$, denote the intervals on which $T$ is continuous, and let $\beta_{i}=T^{\prime}(\xi)$ for any $\xi \in\left(a_{i-1}, a_{i}\right)$.

Let $B=\bigcup_{n=0}^{\infty} T^{-n}\left\{a_{0}, \ldots, a_{8}\right\}$, which is at most a countably infinite set.

For $\xi \in I \backslash B$, define

$$
k_{0}(\xi)=x_{i} \quad \text { if } \xi \in\left(a_{i-1}, a_{i}\right)
$$

and let $k_{i}(\xi)=k_{0}\left(T^{i} \xi\right)$. Then let

$$
\Sigma_{T}=\left\{k(\xi)=\left(k_{i}(\xi)\right)_{i=0}^{\infty}: \xi \in I\right\} \subseteq X=\prod_{n=0}^{\infty}\left\{x_{1}, \ldots, x_{8}\right\} .
$$

As usual, there is a shift operator $\sigma: \Sigma_{T} \rightarrow \Sigma_{T}$ defined by $(\sigma w)_{n}=w_{n+1}$, such that $k(T \xi)=\sigma k(\xi)$.

Define an order $\leqq$ on $X$ as follows. First define

$$
\theta(i)= \begin{cases}1 & \text { if } \beta_{i}>0 \\ -1 & \text { if } \beta_{i}<0\end{cases}
$$

Extend this to finite sequences by

$$
\theta\left(w_{0} w_{1} \cdots w_{m}\right)=\theta\left(w_{0}\right) \theta\left(w_{1}\right) \cdots \theta\left(w_{m}\right) .
$$

Given $w, u \in X$ with $w \neq u$, choose $m$ such that $w_{m} \neq u_{m}$ but $w_{j}=u_{j}$ for $j<m$. Let $w \leqq u$ if

$$
\left(u_{m}-w_{m}\right) \theta\left(w_{0} w_{1} \cdots w_{m}\right)>0,
$$

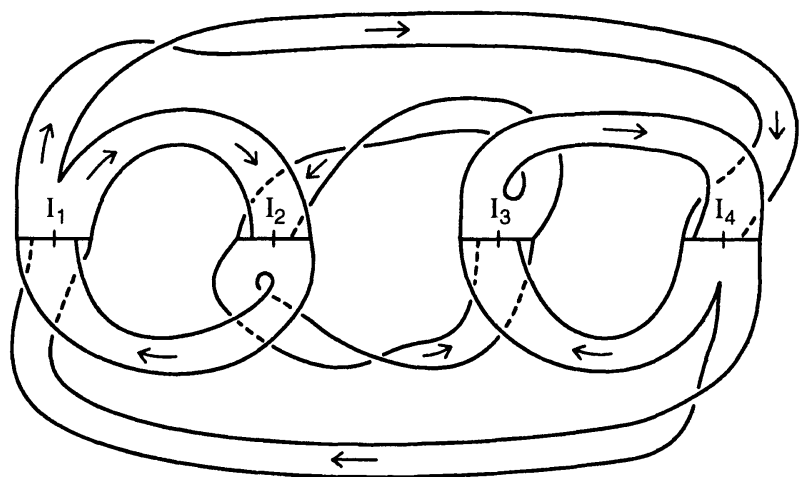

Fig. 8.1. 


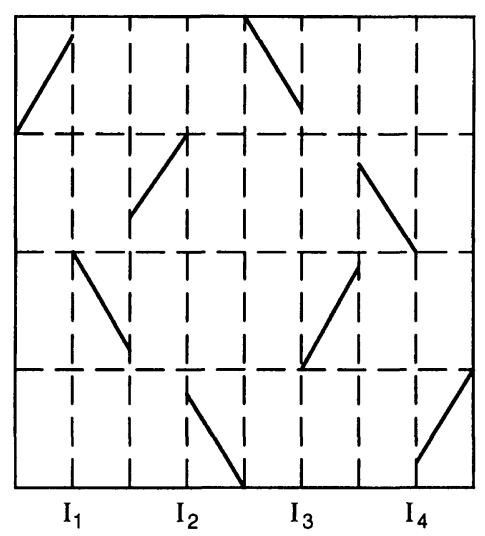

Fig. 8.2.

(taking $\theta(\emptyset)=1$ when $m=0$ ). This order is then the order on $X$ induced by the natural order of the branch line $I$.

It is well known that the limits

$$
u^{(i)}=\lim _{\xi \downarrow a_{l-1}, \xi \notin B} k(\xi) \quad \text { and } \quad v^{(i)}=\lim _{\xi \uparrow a_{i}, \xi \notin B} k(\xi)
$$

exist, (for $1 \leqq i \leqq 8$ ), and that $u^{(i)}, v^{(i)} \in \Sigma_{T}$. (See for example [Bo, p. 37]). These sequences will be called kneading parameters. Further, $\Sigma_{T}$ can be expressed as

$$
\Sigma_{T}=\left\{w \in X: u^{(i)} \leqq \sigma^{k} w \leqq v^{(i)} \quad \text { if } w_{i}=x_{i} \text {, for all } k \geqq 0\right\} .
$$

We define a new template $K$ with semiflow $\Psi_{t}$ and Poincaré map $S: J \rightarrow J$ as illustrated in Fig. 8.3 and Fig. 8.4.

We outline the proof of the following lemma.

Lemma 15. For a suitable choice of Poincaré map $S$, the link of periodic orbits of $\Psi_{t}$ on $K$ is isotopic to a sublink of the periodic orbits of $\Phi_{t}$ on $H_{8}$.

Proof. We describe a sequence of operations which convert $H_{8}$ to $K$.

Modify $T \mid\left(a_{0}, a_{1}\right)$ so that it maps $\left(a_{0}, a_{1}\right)$ linearly into $\left(a_{6}, a_{7}\right)$ with slope $\tilde{\beta}_{1}$, to give a new Poincaré map $T$. We delete the redundant part of the template $H_{8}$. Let

$$
\tilde{u}^{(1)}=\lim _{\xi \downarrow a_{0}} k_{\tilde{T}}(\xi) \quad \text { and } \quad \tilde{v}^{(1)}=\lim _{\xi \uparrow a_{1}} k_{\tilde{T}}(\xi)
$$

be the new kneading parameters. Since $\tilde{\beta}_{1}>0$,

$$
T\left(a_{0}\right)<\tilde{T}\left(a_{0}\right)<\tilde{T}\left(a_{1}\right)<T\left(a_{1}\right),
$$

and hence

$$
u^{(1)}<\tilde{u}^{(1)}<\tilde{v}^{(1)}<v^{(1)} .
$$

Thus from (8.1), we have $\Sigma_{\tilde{T}} \subset \Sigma_{T}$.

We now repeat this operation on the pairs of intervals $\left(a_{6}, a_{7}\right)$ and $\left(a_{5}, T\left(a_{6}^{+}\right)\right)$, $\left(a_{2}, a_{3}\right)$ and $\left(a_{4}, a_{5}\right),\left(a_{4}, a_{5}\right)$ and $\left(a_{7}, a_{8}\right)$, to obtain the template $K^{\prime}$ illustrated in Fig. 8.5, together with the kneading space $\tilde{\Sigma} \subset \Sigma_{T}$. 


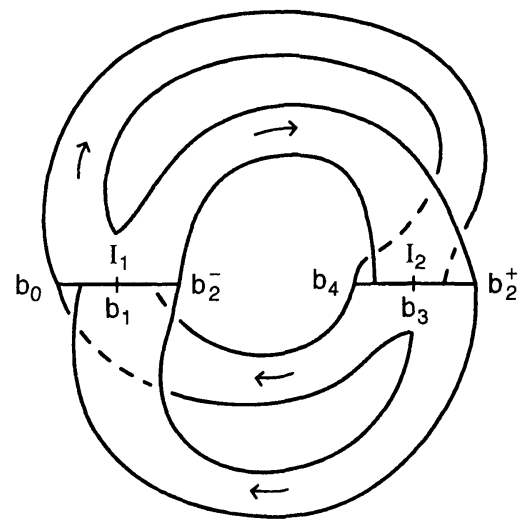

Fig. 8.3.

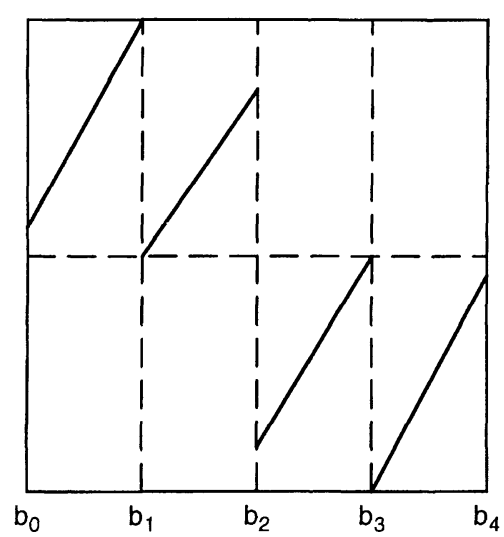

Fig. 8.4.

It is easy to see that the template $K^{\prime}$ can be isotoped to the template $K$. Since all Poincare maps were chosen to be piecewise linear, the Poincaré map on $I_{1} \cup I_{2}$ takes the form illustrated in Fig. 8.4. (Note that it may not be possible to ensure that $\left|S^{\prime}\right|>1$.)

Finally, since the kneading sequences, together with the ordering determine the links of periodic orbits on $K^{\prime}$ and $H_{8}$, it follows from $\tilde{\Sigma} \subset \Sigma_{T}$ that the link of periodic orbits on $K^{\prime}$ is isotopic to a sublink of the periodic orbits on $H_{8}$.

Let $\tilde{\beta}_{1}, \tilde{\beta}_{7}, \tilde{\beta}_{3}, \tilde{\beta}_{5}$ denote the slopes of the modified Poincare map on the intervals $\left(a_{0}, a_{1}\right),\left(a_{6}, a_{7}\right),\left(a_{2}, a_{3}\right)$, and $\left(a_{4}, a_{5}\right)$ respectively, as in the proof of Lemma 15. To ensure that $\left|S^{\prime}\right|>1$, it is sufficient to suppose that $\left|\tilde{\beta}_{1} \tilde{\beta}_{7} \beta_{6}\right|>1$ and $\left|\tilde{\beta}_{3} \tilde{\beta}_{5} \beta_{8}\right|>1$. We shall always assume this.

The standard Lorenz attractor $H_{L}$ or left handed Lorenz attractor, is as illustrated in Fig. 1.1. A right-handed Lorenz attractor $H_{L}^{\prime}$ is defined to be the mirror image of a left-handed Lorenz attractor, (i.e. the $y$-arm crosses over the $x$-arm at the branch line $I$ ).

Lemma 16. The set of periodic orbits of $\Psi_{t}$ on $K$ contains the composite of an arbitrary left-handed with an arbitrary right-handed Lorenz knot, (the lefthanded (respectively right-handed) Lorenz attractor having kneading invariant $\kappa_{1}$ (respectively $\left.\kappa_{2}\right)$ ).

Proof. We observe that the proof of Proposition 6.1 in [BW2], which concerned a specific choice of $S$, can be applied to arbitrary Poincaré maps $S$.

We call the pair of (left- and right-handed) Lorenz templates constructed in Lemma 16 the Lorenz components of $\left(H_{8}, \Phi_{t}\right)$.

From now on, let $p o\left(H_{8}, \Phi_{t}\right)$ denote the link of all periodic orbits of the semiflow $\Phi_{t}$ on $H_{8}$. We use a similar notation for the other templates. Let $\left(\rho_{1}\right)_{t},\left(\rho_{2}\right)_{t}$ be semiflows on $H_{L}, H_{L}^{\prime}$ with their respective kneading invariants $\kappa_{1}, \kappa_{2}$.

Theorem 5. Suppose that the kneading invariants $\kappa_{1}, \kappa_{2}$ given in Lemma 15 are prime and satisfy $\kappa_{1}, \kappa_{2} \in K_{0}$. Then there exist positive constants $M, N$ such that

$$
\#\{\tau: g(\tau) \leqq m\} \gg M \frac{e^{N \sqrt{m}}}{\sqrt{m}},
$$




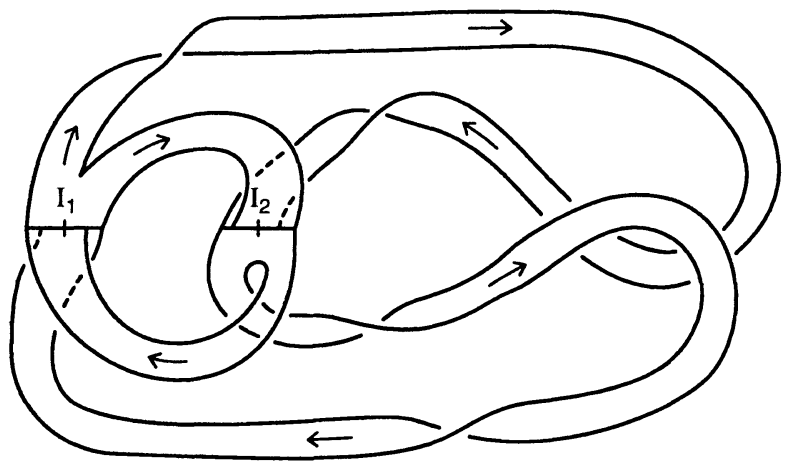

Fig. 8.5.

where $\tau$ denotes a generic closed orbit of $\Phi_{t}$. (Explicitly,

$$
M=\frac{e^{\lambda_{1}\left(1+\frac{1}{2 d_{1}}\right)+\lambda_{2}\left(1+\frac{1}{2 d_{2}}\right)}}{2\left(e^{\lambda_{1}}-1\right)\left(e^{\lambda_{2}}-1\right) \sqrt{d_{1} d_{2}}}
$$

and

$$
N=\sqrt{2}\left(\frac{\lambda_{1}}{\sqrt{d_{1}}}+\frac{\lambda_{2}}{\sqrt{d_{2}}}\right),
$$

where $\lambda_{i}, d_{i}$ are the constants associated to the Lorenz components of $\left(H_{8}, \Phi_{t}\right)$, with kneading invariants $\kappa_{i}$, by Theorem 2.)

Proof. By Lemma 15, there is a sublink $L \subseteq p o\left(K, \Psi_{t}\right)$ such that $L$ consists precisely of all sums $\tau_{1}+\tau_{2}$, where $\tau_{1} \in p o\left(H_{L},\left(\rho_{1}\right)_{t}\right)$ and $\tau_{2} \in p o\left(H_{L}^{\prime},\left(\rho_{2}\right)_{t}\right)$. Since each right-handed Lorenz attractor is the mirror image of a left-handed Lorenz attractor, and a knot and its mirror image have the same genus, Theorem 2 holds for righthanded Lorenz attractors. Also, note that if $\tau=\tau_{1}+\tau_{2}$ then $g(\tau)=g\left(\tau_{1}\right)+g\left(\tau_{2}\right)$,

$$
\#\left\{\tau \in p o\left(H_{8}, \Phi_{t}\right): g(\tau) \leqq m\right\} \geqq \#\left\{\tau \in p o\left(K, \Psi_{t}\right): g(\tau) \leqq m\right\}
$$

by Lemma 15 ,

by Lemma 16 ,

$$
\begin{gathered}
\geqq \#\left\{\tau \in p o\left(K, \Psi_{t}\right): \tau=\tau_{1}+\tau_{2}, \tau_{1} \in p o\left(H_{L},\left(\rho_{1}\right)_{t}\right),\right. \\
\left.\tau_{2} \in p o\left(H_{L}^{\prime},\left(\rho_{2}\right)_{t}\right), g\left(\tau_{1}+\tau_{2}\right) \leqq m\right\}
\end{gathered}
$$

$$
\begin{gathered}
\geqq \#\left\{\tau \in p o\left(K, \Psi_{t}\right): \tau=\tau_{1}+\tau_{2}, \tau_{1} \in p o\left(H_{L},\left(\rho_{1}\right)_{t}\right),\right. \\
\left.\tau_{2} \in p o\left(H_{L}^{\prime},\left(\rho_{2}\right)_{t}\right), g\left(\tau_{1}\right)+g\left(\tau_{2}\right) \leqq m\right\} \\
\geqq \#\left\{\tau_{1} \in p o\left(H_{L},\left(\rho_{1}\right)_{t}\right): g\left(\tau_{1}\right) \leqq(1 / 2) m\right\} \\
\quad \#\left\{\tau_{2} \in p o\left(H_{L}^{\prime},\left(\rho_{2}\right)_{t}\right): g\left(\tau_{2}\right) \leqq(1 / 2) m\right\} .
\end{gathered}
$$

Applying Theorem 2 to (8.3) gives (8.2).

Acknowledgements. This paper is a revised and extended version of a chapter of my $\mathrm{Ph}$.D. thesis at the University of Warwick. I would like to thank my supervisor Professor Bill Parry for starting me 
off on such an interesting topic. Also, thanks to Professor Peter Walters and Dr. Maurice Dodson for their comments on an earlier draft of this paper. The preparation of this paper was completed at the SFB 170, Göttingen, whom I would like to thank for their hospitality and support.

\section{References}

[A] Abramov, L.M.: On the entropy of a flow. Dokl. Akad. Nauk. SSSR 128, 873-875 (1959)

[BK] Baladi, V., Keller, G.: Zeta functions and transfer operators for piecewise monotonic transformations. Commun. Math. Phys. 127, 459-477 (1990)

[Bi] Birman, J.S.: Braids, links and mapping class groups. Ann. Math. Studies 81, Princeton, NJ: Princeton University Press, 1974

[Bo] Bowen, R.: On Axiom A diffeomorphisms. Conf. Board Math. Sciences Regional Conferences Series 35, 1977

[BW1] Birman, J.S., Williams, R.F.: Knotted periodic orbits in dynamical systems I: Lorenz's equations. Topology 22, 47-82 (1983)

[BW2] Birman, J.S., Williams, R.F.: Knotted periodic orbits in dynamical systems II: Knot holders for fibred knots. Contemp. Math. 20, 1-60 (1983)

[FW] Franks, J., Williams, R.F.: Braids and the Jones polynomial. Trans. Am. Math. Soc. 303, 97-108 (1987)

[G] Glendinning, P.: The structure of $\beta$-transformations and conjugacies to Lorenz maps. University of Warwick, Preprint, 1987

[Gu] Guckenheimer, J.: A strange, strange attractor. Lecture notes in Appl. Math. Sci. 19, 368-391 (1976)

[Lo] Lorenz, E.N.: Deterministic non-periodic flows. J. Atmospheric Sci. 20, 130-141 (1963)

[P1] Parry, W.: Symbolic dynamics and transformations of the unit interval. Trans. Am. Math. Soc. 122, 368-378 (1966)

[P2] Parry, W.: An analogue of the Prime Number Theorem for shifts of finite type and their suspensions. Isr. J. Math. 45, 41-52 (1983)

[P3] Parry, W.: The Lorenz attractor and a related population model. In: Ergodic Theory, Lecture Notes in Mathematics 729, eds. Dold A., Eckmann B., Berlin, Heidelberg, New York: Springer, 1979

[P4] Parry, W.: Intrinsic Markov chains. Trans. Am. Math. Soc. 112, 55-65 (1965)

[P5] Parry, W.: Bowens's equidistribution theory and the Dirichlet Density Theorem. Ergod. Th. and Dyn. Syst. 4, 117-134 (1984)

[PP] Parry, W., Pollicott, M.: Zeta functions and the periodic orbit structure of hyperbolic dynamics. Asterisque 187-188, Publ. Math. Soc. France, 1990

[R] Ruelle, D.: The Lorenz attractor and a problem of turbulence. In: Turbulence and Navier Stokes Equations, L.M.N. Vol. 565, Berlin, Heidelberg, New York: Springer, 1976

[RT] Ruelle, D., Takens, F.: On the nature of turbulence. Commun. Math. Phys. 20, 167 (1971)

[Wa] Waddington, S.: The prime orbit theorem for quasihyperbolic toral automorphisms. Mh. für Math. 112, 235-248 (1991)

[W1] Williams, R.F.: The structure of the Lorenz attractor. Publ. I.H.E.S. 50, 321-347 (1979)

[W2] Williams, R.F.: The bifurcation space of the Lorenz attractor. Ann. New York Acad. Sci. 316, 393-399 (1979)

[W3] Williams, R.F.: A new zeta function, natural for links. University of Texas at Austin, Preprint, 1991

[W4] Williams, R.F.: Survey lecture: Geometric theory of dynamical systems. In: Dynamical Systems. Pittman Research Notes in Mathematics No. 221, Longman, 1990 
